\title{
Ramping turn-to-turn loss and magnetization loss of a No-Insulation (RE)Ba2Cu3Ox high temperature superconductor pancake coil
}

\author{
Y. Wang, ${ }^{1,2)}$ H. Song, ${ }^{3, a)}$ W. Yuan, ${ }^{2)}$ Z. Jin,,${ }^{1)}$ and Z. Hong, ${ }^{1)}$ \\ ${ }^{1}$ Department of Electrical Engineering, Shanghai Jiao Tong University, Shanghai,200240, China. \\ ${ }^{2}$ Department of Electronic and Electrical Engineering, University of Bath, Bath, BA27AY, UK \\ ${ }^{3}$ Brookhaven National Laboratory, Upton, NY, 11973, USA.
}

This paper is to study ramping turn-to-turn loss and magnetization loss of a no-insulation (NI) high temperature superconductor (HTS) pancake coil wound with (RE)Ba2Cu3Ox (REBCO) conductors. For insulated (INS) HTS coils, a magnetization loss occurs on superconducting layers during a ramping operation. For the NI HTS coil, additional loss is generated by the 'bypassing' current on the turn-to-turn metallic contacts, which is called "turn-toturn loss" in this study. Therefore, the NI coil's ramping loss is much different from that of the INS coil, but few studies have been reported on this aspect. To analyze the ramping losses of NI coils, a numerical method is developed by coupling an equivalent circuit network model and a $\mathrm{H}$-formulation finite element method (FEM) model. The former model is to calculate NI coil's current distribution and turn-to-turn loss, the latter model is to calculate the magnetization loss. A test NI pancake coil is wound with REBCO tapes and the reliability of this model is validated by experiments. Then the characteristics of the NI coil's ramping losses are studied using this coupling model. Results show that the turn-to-turn loss is much higher than the magnetization loss. The NI coil's total ramping loss is much higher than that of its insulated counterpart, which has to be considered carefully in the design and operation of NI applications. This paper also discusses the possibility to reduce NI coil's ramping loss by decreasing the ramping rate of power supply or increasing the coil's turn-to-turn resistivity.

\section{INTRODUCTION}

Quench protection has always been a critical challenge for high temperature superconductor (HTS) coils and magnets ${ }^{1-3}$. To enhance thermal stability, winding the HTS coil directly without any turn-to-turn insulations has been discussed and experimented ${ }^{4-6}$. During a quench, transport current can bypass the local normal region through metallic turn-to-turn contacts, therefore, the NI coil shows an enhanced thermal stability and self-protection features compared to its insulated counterpart ${ }^{4}, 5,7-12$. It is promising to apply the NI technique on the high field magnet (MRI/NMR), accelerator magnet and power application devices 9, 10,13-15. On the other hand, due to the absence of turn-to-turn electrical insulation, the transport current can flow through turn-to-turn contacts during a time-varying operation. This leads to an intrinsic delay in the ramping process of NI coils, which has been studied in

a) Email: honghaisong@gmail.com. 
previous publications ${ }^{16-21}$. Moreover, it generates extra losses on the turn-to-turn contacts during the ramping operation, which seems another challenge for the NI coils.

The total loss generated in the ramping process is an important issue for superconducting coils and magnets, which needs to be precisely studied to predict and reduce the refrigeration requirements of applications ${ }^{22-27}$. For the conventionally insulated HTS coils, a magnetization loss are generated because of flux creep and flux jump in superconductors during the ramping operation, which is the main part of the traditional AC loss ${ }^{24,28-31}$. For the NI HTS coils, an extra Joule loss is generated across the turn-to-turn contacts by the radial current besides the conventional magnetization loss in superconductors, which is called "turn-to-turn loss" in this study. Therefore, the ramping loss of the NI coil is very different from that of its insulated counterpart. However, very few studies have investigated this loss phenomenon.

This paper is to study the ramping losses of NI pancake coils wound with REBCO tapes. A numerical method has been developed by coupling an equivalent circuit network model and a finite element method (FEM) model based on H-formulation. The equivalent circuit model is to calculate the turn-to-turn loss and the FEM model is to calculate the magnetization loss in superconducting layers. A test NI pancake coil is wound with REBCO tapes and charging experiments are performed on this coil. The ramping losses from simulations and measurements are compared so as to validate the numerical model. Then, the characteristics of NI coil's ramping losses are analyzed in detail using this model. The difference between NI coil and INS coil is compared in more detail. Discussions are performed on the measures to reduce the ramping loss of NI coils: decreasing ramping rate and increasing turn-toturn resistivity.

\section{NUMERICAL METHOD}

In the ramping process of NI REBCO pancake coils, part of transport current 'bypasses' through turn-to-turn contacts along the radial direction, which is called 'radial current' for clarity. The other part of current flowing along the azimuthal direction is called 'azimuthal current'. Joule losses are generated by the radial current across turn-toturn resistances. Magnetization loss is generated in type-II superconductors due to the flux creep and flux moving in the superconducting layers. During a successful ramping operation, the transport current is often below critical current, most of the azimuthal current flows in the superconducting layers ${ }^{32}$. Therefore, the total ramping loss of NI coils consists of the turn-to-turn loss and magnetization loss, which can be expressed as follows:

$$
Q_{\text {ramp }}=Q_{t 2 t}+Q_{s c}
$$

where $Q_{\text {ramp }}, Q_{t 2 t}$, and $Q_{s c}$ represents the total ramping losses, turn-to-turn loss and magnetization loss respectively.

To calculate the ramping losses, a numerical method is built for the NI REBCO pancake coil, as shown in Fig. 1. The model couples an equivalent circuit network model and a FEM model based on $\mathrm{H}$-formulation. The equivalent circuit network model is to calculate the current distribution inside the NI coil, and the turn-to-turn losses generated by radial currents can be obtained directly from this model. The current distribution from the equivalent circuit model is inputted into the FEM model to calculate the magnetization loss in superconducting layers. The two models are implemented and solved simultaneously by MATLAB codes and a time dependent solver in Comsol 
Multiphysics $^{\mathrm{TM}}$. In this study, assuming that the cooling power is effectively enough, thus these losses do not result in a considerable temperature rise.

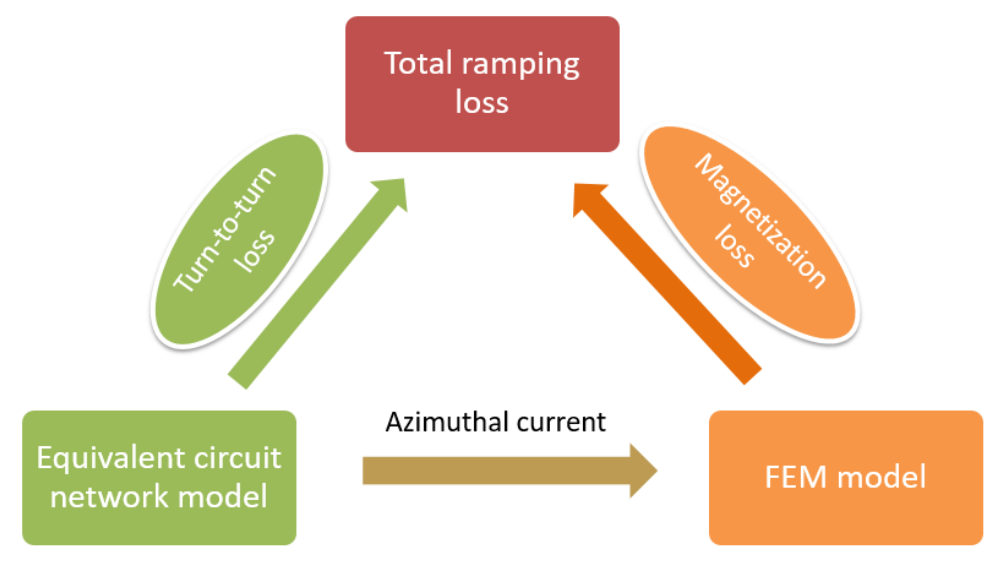

FIG. 1. The numerical algorithm to calculate the ramping losses of NI HTS coils.

\section{A. Equivalent circuit network model}

In the equivalent circuit network model, each turn of the coil is subdivided into fine arc elements, each arc element is equivalent to circuit parameters, so that the whole coil is equivalent to a circuit network ${ }^{32-34}$. FIG. 2(a) shows the schematic illustration of the model for the NI pancake coils, in which each turn is subdivided into 4 arc elements [7, 28]. In the following simulations, each turn will be subdivided into 48 arc elements. At each independent circuit node, the governing equation can be derived from Kirchhoff's current law (KCL); in each independent circuit mesh, the governing Eq. is derived from Kirchhoff's voltage law (KVL):

$$
\left\{\begin{array}{l}
\sum_{k} i_{k}+\sum_{k} j_{k}=0 \\
\sum_{m=1}^{4} u_{m, k}=0
\end{array}\right.
$$

where $i_{k}$ and $j_{k}$ represent the azimuthal and radial current of the $k$-th arc element respectively. $u_{m, k}$ is the voltage on the $m$-th circuit branch of the $k$-th independent circuit mesh. More details about this model have been introduced in previous publications, in which the model has been validated by the charging and discharging experiments ${ }^{32}$.

The turn-to-turn loss energy $Q_{t 2 t}$ generated by radial current can be calculated by:

$$
\left\{\begin{array}{l}
W_{t 2 t}=\sum_{k} j_{k}^{2} R_{k} \\
Q_{t 2 t}=\int W_{t 2 t} d t
\end{array}\right.
$$

where $W_{t 2 t}$ is the Joule loss power on turn-to-turn contacts, $R_{k}$ is the equivalent turn-to-turn resistance of the $k$-th arc element. Here, the turn-to-turn resistance $R_{k}$ is an equivalent resistance including that of the turn-to-turn contact, normal and superconducting layers along the radial direction. 


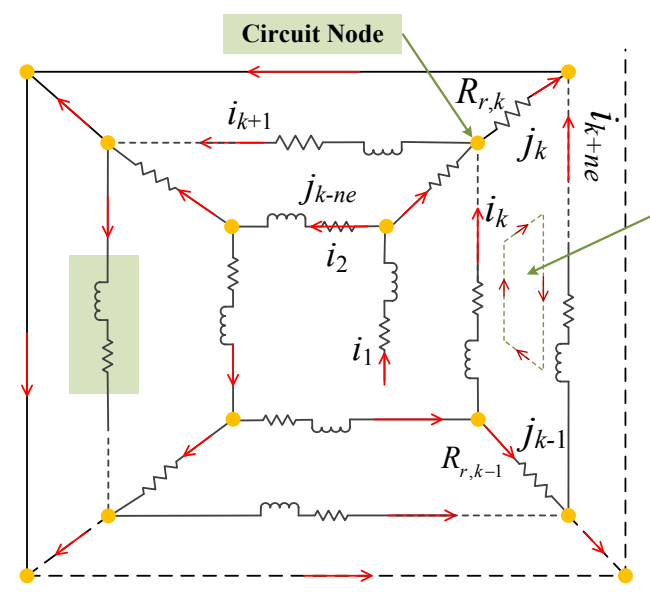

(a) Equivalent circuit network model

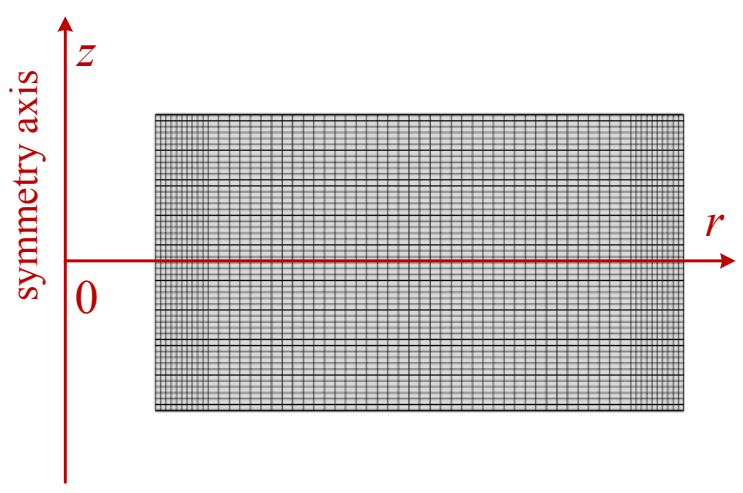

(b) FEM model

FIG. 2. The schematic illustration of the equivalent circuit network model and FEM model for NI REBCO coil.

\section{B. FEM model based on H-formulation}

The FEM model based on $\mathrm{H}$-formulation is to calculate the magnetization loss in superconductors. Previous studies have shown that the radial current inside the NI coil presents a uniform distribution along angular direction in the charging and discharging process (due to the axis symmetry). The magnetic field induced by the radial current is cancelled with each other. The field of the NI coil is contributed mainly by the azimuthal current [32]. Therefore, only the azimuthal current is needed in the calculation of magnetization loss. Previous studies have also shown that the azimuthal current on most of turns presents a uniform distribution along the angular direction, except for several turns near current leads ${ }^{32,34}$. Therefore, to reduce the computation cost, a 2D axisymmetric FEM model is used for the calculation of magnetization loss, as shown in Fig. 2(b).

In this study, it employs the H-formulation as the governing equation of the FEM model, which is an effective numerical approach to simulate the electromagnetic behaviors of HTS $28,30,31,35-37$. The governing Eq. is derived from Maxwell's equation:

$$
\left\{\begin{array}{l}
\nabla \times \mathbf{E}=-\mu \frac{\partial \mathbf{H}}{\partial t} \\
\nabla \times \mathbf{H}=\mathbf{J}
\end{array}\right.
$$

where $\mathbf{E}$ represents the electrical field, $\mathbf{H}$ represents magnetic field intensity respectively. $\mathbf{J}$ represents the current density, which is the azimuthal current density from the above the equivalent circuit network model. In this 2D axisymmetric model, the average value of the azimuthal current along turns is used, which is calculated by ${ }^{19,32}$ :

$$
i_{\text {turn }, k}=\sum_{m=(k-1) n_{e}+1}^{k n_{e}} i_{m} / n_{e}
$$

where $i_{\text {turn, }, k}$ represents the average azimuthal current on the $k$-th turn. $i_{m}$ is the azimuthal current of $m$-th arc element, which is from the above network model. $n_{e}$ is the number of arc elements in each turn. Integral constraints are imposed on each turn to ensure the transport current: 


$$
i_{\text {turn }, k}(t)=\int_{\Omega_{k}} J_{k}(r, z, t) d \Omega ; k \in\left\{1,2, \ldots, N_{t}\right\}
$$

where $J_{k}(r, z, t)$ is current density in superconducting layers. $\Omega_{k}$ is the cross section domain of the $k$-th turn. $N_{t}$ is the number of turns.

For HTS materials, the highly nonlinear relationship between current and the voltage can be expressed: ${ }^{38}$ :

$$
\mathbf{E}=E_{0}\left(\frac{\mathbf{J}}{J_{c}(\mathbf{B})}\right)^{n} \frac{\mathbf{J}}{|\mathbf{J}|}
$$

where $E_{0}=1 \mu \mathrm{V} / \mathrm{cm}, n$ presents the sharpness from superconducting state to normal state, it is set to be 40 in this simulation ${ }^{39} . J_{c}$ is critical current density, whose anisotropic field dependence can be expressed as following Eq.$^{40}$ :

$$
J_{c}(\mathbf{B})=\frac{J_{c 0}}{\left[1+\sqrt{\left(k B_{p a r}\right)^{2}+B_{p e r}^{2}} / B_{c}\right]^{b}}
$$

where $J_{c 0}$ is the critical current density in self-field, $B_{p a r}$ and $B_{p e r}$ are the magnetic field parallel and perpendicular to the tape surface respectively. $k, b, B_{c}$ is the shape parameter, which is obtained by fitting experimental data. The REBCO tape used in this study is from SuNAM, Korea, whose shape parameter is estimated to be $k=0.05869$ 、 $b=0.7636 、 B_{c}=101.7 \mathrm{mT}$.

Therefore, the magnetization loss in superconducting layers can be calculated by the following integral:

$$
\left\{\begin{array}{l}
W_{s c}=\int_{\Omega} \mathbf{E} \cdot \mathbf{J} \mathrm{d} \Omega \\
Q_{s c}=\int W_{s c}(t) \mathrm{dt}
\end{array}\right.
$$

where $W_{s c}$ is the magnetization loss power and $Q_{s c}$ is the total magnetization loss energy in the whole ramping process.

The REBCO tape consists of superconducting layer and several metallic layers. The thickness of the superconducting layer is less than $1 \%$ of the tape's total thickness. Since most of the azimuthal current flows in the superconducting layer below critical current, the normal layers can be neglected and the superconducting layer can be enlarged to the whole domain of the tape. To reduce the computation cost, Zermeno et al developed the Hformulation FEM model and proposed a homogenization method, in which the current density in superconducting layer is assumed to be homogenous along the direction perpendicular to the tape surface. This numerical method shows a fast computation and convergence and it is very suitable for large scale HTS applications. More details about this method have been shown in previous publications ${ }^{41-43}$. The current distribution near the inner and outer radii of the coil varies considerably, so these areas are meshed finer than the others, as shown in Fig. 2(b). This homogenization FEM model based on $\mathrm{H}$-formulation can conduct an accurate calculation on the AC loss of REBCO tapes, which is the magnetization loss in this study. The AC loss from this model shows a good agreement with that from experiments ${ }^{41}$. Therefore, it is chosen to calculate the magnetization loss of REBCO coil in this study. A time dependent solver from COMSOL Mutiplphysics ${ }^{\mathrm{TM}}$ is used to solve this model. 


\section{EXPERIMENTAL VALIDATION}

A double pancake (DP) coil has been wound by REBCO tapes to validate the above numerical model. The coil is wound directly without any insulation and a constant tension $39 \mathrm{~N}$ is applied in the winding process. The tape has brass lamination and it is produced by SuNAM, Korea. The length and thickness of the tape are $4.2 \mathrm{~mm}$ and 0.25 $\mathrm{mm}$ respectively. The critical current of the tape in self-field is $170 \mathrm{~A}$ at $77 \mathrm{~K}$. The coil is tested in liquid nitrogen $(77 \mathrm{~K})$ and its critical current is $97 \mathrm{~A}$; the equivalent turn-to-turn resistivity is measured at $28 \mu \Omega \cdot \mathrm{cm}^{2}{ }^{32}$. More specifications about the coil are shown in Table I.

This test NI coil is charged to $60 \mathrm{~A}$ with a ramping rate of $0.88 \mathrm{~A} / \mathrm{s}$. A hall probe is set at the coil center to measure the magnetic field induced. Fig. 3 shows the variation of the transport current from power supply $I_{o p}$, coil voltage $U_{\text {coil }}$ and magnetic field $B_{c}$ in the charging process. A significant delay between transport current and magnetic field at coil center occurs as expected. Finally, the field increases up gradually to the desired value. The charging process of NI coils ends when the field induced is ramped up to the desired value in a steady state. The ramping losses of the NI coil include all the losses in the whole ramping process.

The total energy inputted into the NI coil in whole the ramping process $Q_{\text {input }}$ consists of two parts: one is stored up in the coil as magnetic field energy $Q_{m}$, the other one is dissipated as ramping losses $Q_{\text {ramp }}$. Therefore, the ramping loss can be calculated as:

$$
Q_{\text {ramp }}=Q_{\text {input }}-Q_{m}
$$

where $Q_{m}$ is the magnetic energy stored in the coil at the end of the charging process. It is calculated by the following Eq.:

$$
Q_{m}=\int_{\Omega} \mathbf{B} \cdot \mathbf{H d} \Omega
$$

For the NI coil in this test, the $Q_{m}$ is $14.5 \mathrm{~J}$ when it is charged to $60 \mathrm{~A}$ in a steady station. The total energy inputted into the NI coil $Q_{\text {input }}$ can be calculated by the following Eq.:

$$
Q_{\text {input }}=\int U_{\text {coil }}(t) I_{o p}(t) \mathrm{d} t
$$

where the coil voltage $U_{\text {coil }}$ and transport current $I_{o p}$ is obtained from measurement, as shown in Fig. 3.

Fig. 4 shows the ramping loss from simulations and experiments under different ramping rates of power supply. Its overall agreement between simulations and experiments validates the reliability of the model proposed though difference does exist. The difference is likely due to a few factors. First, a large part of the energy inputted into the NI coil is stored up as magnetic energy, and the ramping loss energy measured accounts for a small proportion of the total injected energy. For the test with a ramping rate of $0.88 \mathrm{~A} / \mathrm{s}$, the total energy inputted into the NI coil $Q_{\text {input }}$ is $23 \mathrm{~J}$, but $14.5 \mathrm{~J}$ is stored as magnetic energy. Therefore, when the ramping loss energy $Q_{\text {ramp }}$ is subtracted from the total energy inputted, all the measurement errors of the total input energy $Q_{i n p u t}$ is passed on to the ramping loss energy $Q_{\text {ramp }}$. This likely results in larger error percentage for the ramping loss energy achieved in the experimental measurements ${ }^{44,45}$. Second, in the experiments, the current from power supply is ramped up approximately linearly in the whole ramping process, but in each fine step, the current is ramped up discreetly with a rectangle latter shape, which comes from the power supply operating mode. In the simulation, the transport current is ramped up perfectly linearly, which has the same linear ramping rate with the experiments in the whole ramping process. Third, the $I_{c}-\mathbf{B}$ 
curves used in this simulation may have some errors in its fitting function, which can also lead to some errors on the magnetization loss simulations and experiments. The other possible cause of the discrepancy is that the actual turnto-turn resistivity is somewhat non-uniform due to varying turn-to-turn pressure which may be accumulated from the winding, cooling and energization process. Meanwhile the relationship between the resistivity and pressure is not clear which may depend on temperature, contact surface condition, longitudinal strain, et al. Such study is being performed and will be reported in the near future, but not be included here.

TABLE I. Specification of the test NI coil and sample NI coil.

\begin{tabular}{ccc}
\hline \hline Parameters & Test coil & Sample coil \\
\hline Coil type & DP & SP \\
Number of turns & $62 \times 2$ & 100 \\
Inner/outer diameter & $245 / 276 \mathrm{~mm}$ & $100 / 140 \mathrm{~mm}$ \\
Total length of wire & $101 \mathrm{~m}$ & $38 \mathrm{~m}$ \\
Coil inductance, cal. & $8.11 \mathrm{mH}$ & $1.886 \mathrm{mH}$ \\
$B_{z}$ per amp & $0.59 \mathrm{mT} / \mathrm{A}$ & $4 \mathrm{mT} / \mathrm{A}$ \\
$I_{c}$ coil & $97 \mathrm{~A} @ 77 \mathrm{~K}$ & $115 \mathrm{~A} @ 77 \mathrm{~K}$ \\
Turn-to-turn resistivity & $28 \mu \Omega \cdot \mathrm{cm}^{2}$ & $10 \sim 100 \mu \Omega \cdot \mathrm{cm}^{2}$ \\
\hline \hline
\end{tabular}

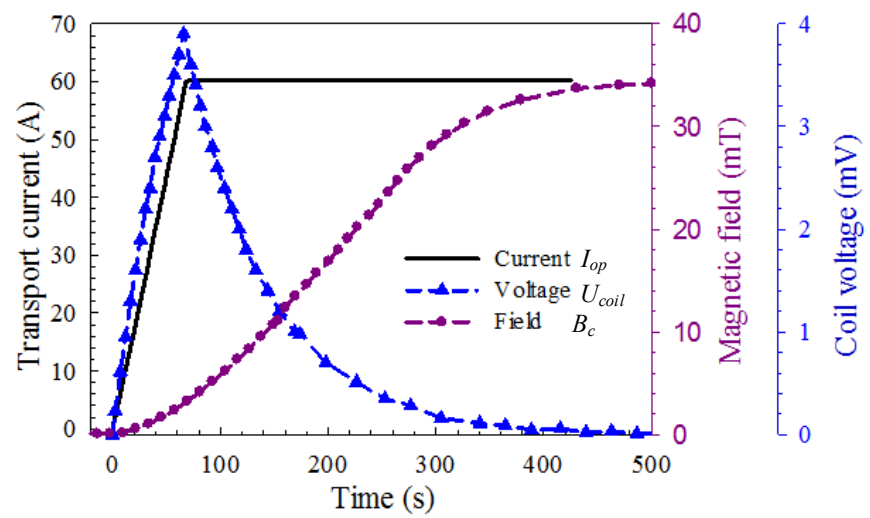

FIG. 3. The measured transport current, coil voltage and magnetic field at coil centre in a ramping process of the test coil.

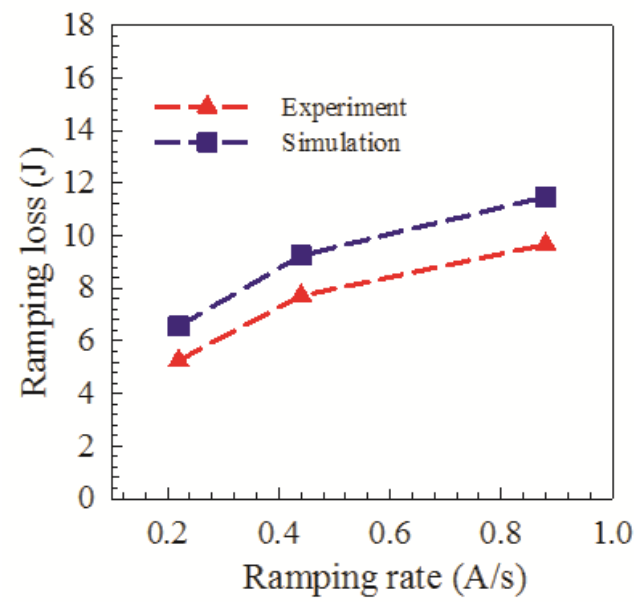

FIG. 4. The ramping losses of the test NI coil from simulations and experiments. 


\section{RAMPING LOSSSES OF NI COILS}

This section is to study the characteristics of NI coils' ramping loss using the above numerical model. The difference between the NI coil and its insulated counterpart are also compared. A sample single pancake (SP) NI coil and its insulated counterpart are designed to conduct this study. The coil is wound with REBCO tapes from SuNAM, Korea. The width and thickness of the tape are $4.0 \mathrm{~mm}$ and $0.2 \mathrm{~mm}$ respectively. The critical current of the tape at $77 \mathrm{~K}$ is $230 \mathrm{~A}$ in self-field and its field dependence is expressed as Eq. (8). More details about this sample coil are shown in Table I. Simulations are performed on the sample NI coil using the above numerical model, in which the transport current from the power supply is ramped up to $80 \mathrm{~A}$ with a ramping rate of $5 \mathrm{~A} / \mathrm{s}$. This coil's equivalent turn-to-turn resistivity is set to be $30 \mu \Omega \cdot \mathrm{cm}^{2}$ in this study.

\section{A. Turn-to-turn and magnetization loss}

Fig. 5 shows the turn-to-turn loss power $W_{t 2 t}$, average radial current of the whole coil $I_{r_{-} a v}$ and transport current in the ramping process, where $I_{r_{-} a v}$ is the average value of the radial current flowing through each turn ${ }^{19,32}$. The turn-toturn loss power increases with the transport current, and it reaches a peak point when the transport current increases up to the target current $80 \mathrm{~A}$ at $t=16 \mathrm{~s}$, then it drops rapidly to the zero. The turn-to-turn loss power is generated by the radial current, so the show a same trend. The magnetization loss power $W_{s c}$ shows a much gentler and slower variation than the turn-to-turn loss power, as shown in Fig. 6. The magnetization loss power increases with the transport current, and continues to increase after the transport current stops increasing at $t=16 \mathrm{~s}$. It reaches to the peak value at $t=21 \mathrm{~s}$, then drops slowly to zero. Peak time difference between the turn-to-turn loss power and magnetization loss power is about $4 \mathrm{~s}$, which is likely because the current needs some time to reach critical state equilibrium while the radial current is transforming into the azimuthal current.

Fig.7 illustrates two-dimensional radial distribution of the turn-to-turn loss energy density $Q_{t 2 t}$ and magnetization loss energy density $Q_{s c}$ in the whole ramping process. Note that the tape thickness is enlarged 3 times for a better representation. Both the turn-to-turn loss energy and the magnetization loss energy show a non-uniform distribution among turns. The turn-to-turn loss near the outer side of the coil is much higher than that on the turns near the inner side. The maximum turn-to-turn loss energy density (at $67^{\text {th }}$ turn) is about three times of the minimum value at the $1^{\text {st }}$ turn. Fig. 8 shows the distribution of the radial current density and azimuthal current in the ramping process, which determines the distribution of the turn-to-turn loss energy. Additionally, the distribution of the radial current depends on the turn's electromagnetic coupling with others $[28,30]$. The magnetization loss energy $Q_{s c}$ in middle part of the coil is much higher than that near the inner and outer sections of the coil. The maximum value is about two times of the minimum one. Fig. 9 shows the distribution of the normalized current in the superconductors and magnetic field perpendicular to the tape surface. The middle turns have a higher perpendicular magnetic field and more current penetration regions $\left(J \approx J_{c}\right)$ than other turns, which leads to more magnetization loss.

The peak of turn-to-turn loss power can be about 20 times larger than that of magnetization loss power, as shown in the Fig. 5 and Fig. 6 . The power peak of the turn-to-turn loss is about $300 \mathrm{~mW}$ at turn $67^{\text {th }}$, while the power peak of the magnetization loss is only $6 \mathrm{~mW}$ at around turn $50^{\text {th }}$. The total turn-to-turn loss energy in the whole ramping 
process is $3.63 \mathrm{~J}$, the total magnetization loss energy is only $0.17 \mathrm{~J}$. Therefore, for the NI coil, the turn-to-turn loss accounts for a main proportion of the total ramping loss.

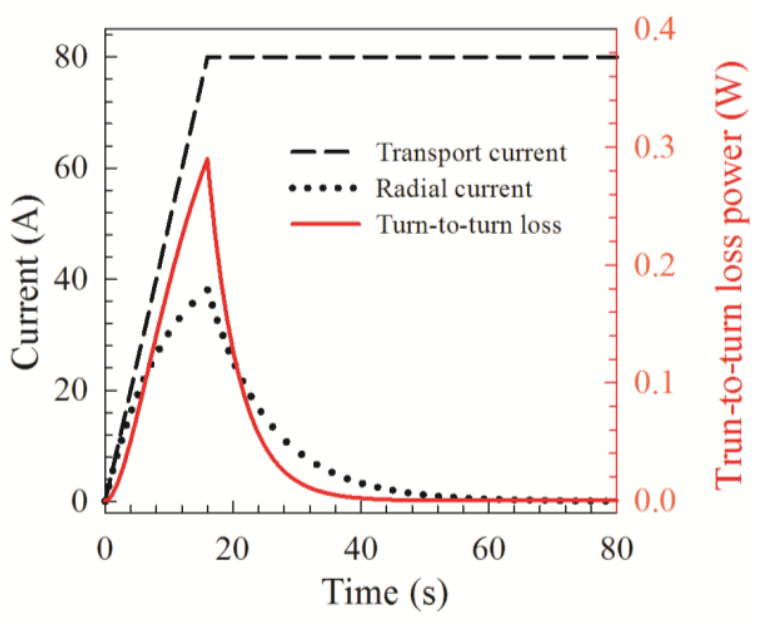

FIG. 5. Turn-to-turn loss power, transport current and average radial current in the ramping process from calculation

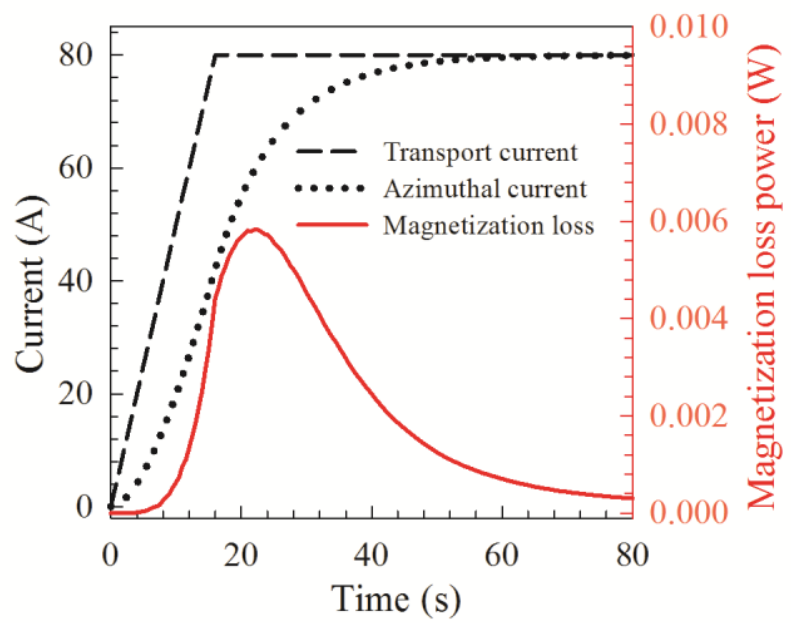

FIG. 6. Magnetization loss power, average azimuthal current in the ramping process from calculation.

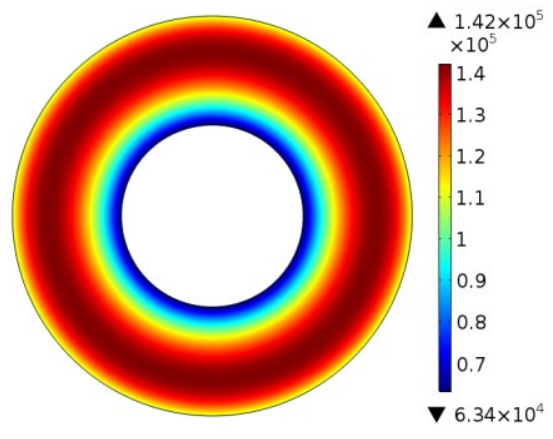

(a) Turn-to-turn loss energy density $\left(\mathrm{J} / \mathrm{m}^{3}\right)$

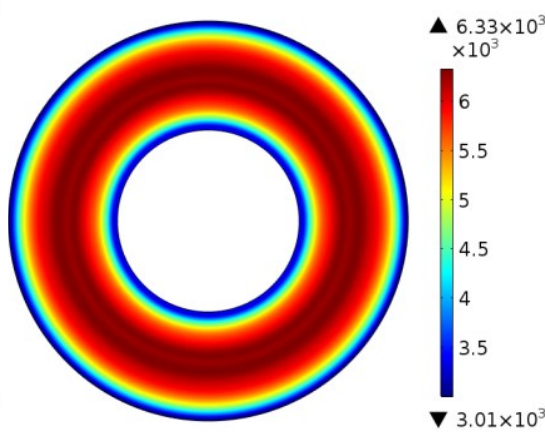

(b) Magnetization loss energy density $\left(\mathrm{J} / \mathrm{m}^{3}\right)$

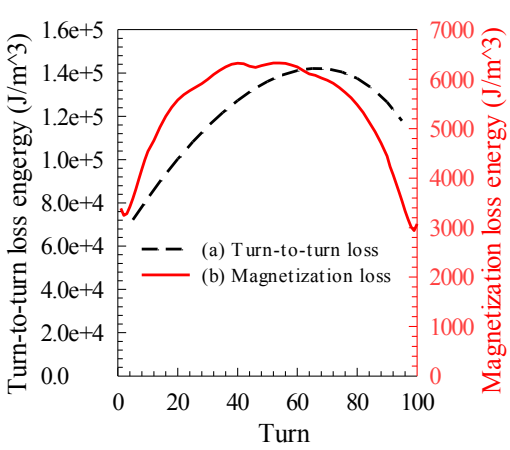

FIG. 7. Distribution of the turn-to-turn loss and magnetization loss energy density in the whole ramping process, and their quantitative density at each turn. 


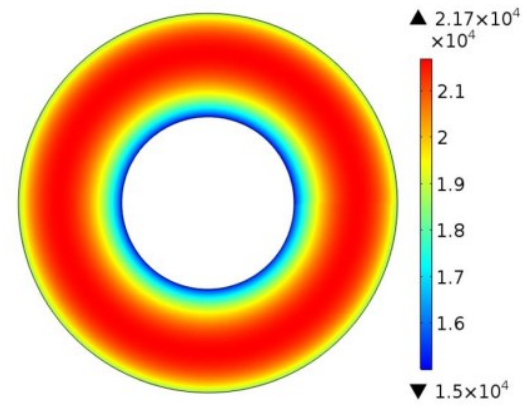

$t=10 \mathrm{~s}$

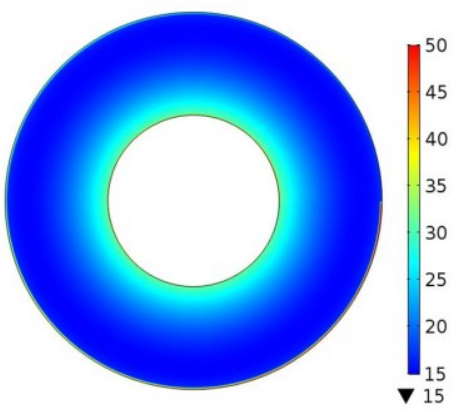

$t=10 \mathrm{~s}$

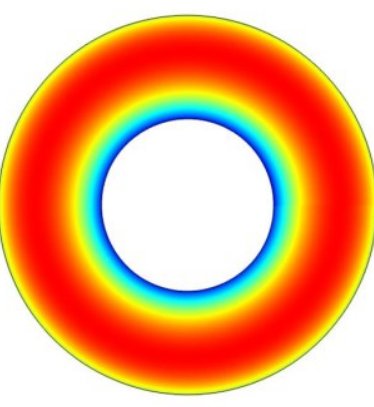

$t=16 \mathrm{~s}$

(a) Radial current density $\left(\mathrm{J} / \mathrm{m}^{3}\right)$

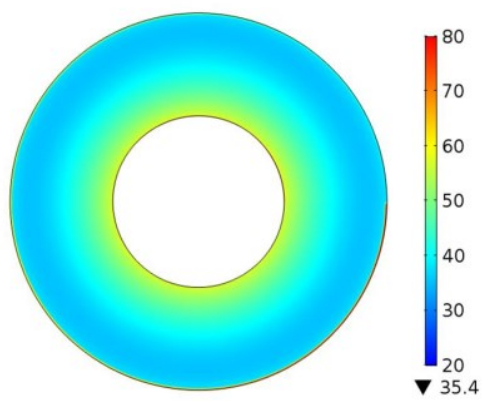

$t=16 \mathrm{~s}$

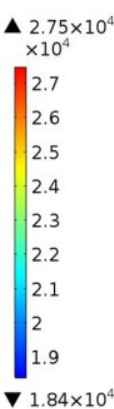

3

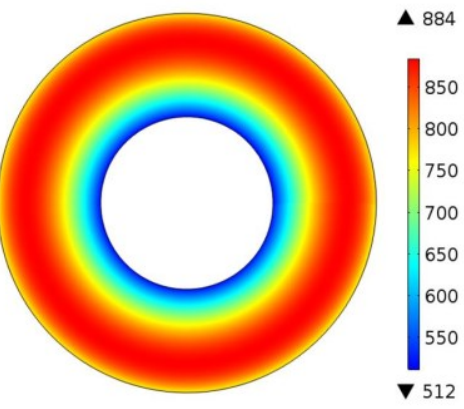

$t=50 \mathrm{~s}$

(b) Azimuthal current (A)

FIG. 8. Distribution of the radial and azimuthal current density $\left(\mathrm{A} / \mathrm{m}^{2}\right)$ in the whole ramping process at three sequential time steps, $t=10 \mathrm{~s}, 16 \mathrm{~s}$ and $50 \mathrm{~s}$. Note that when $t=16 \mathrm{~s}$, the power supply current reaches its maximum.
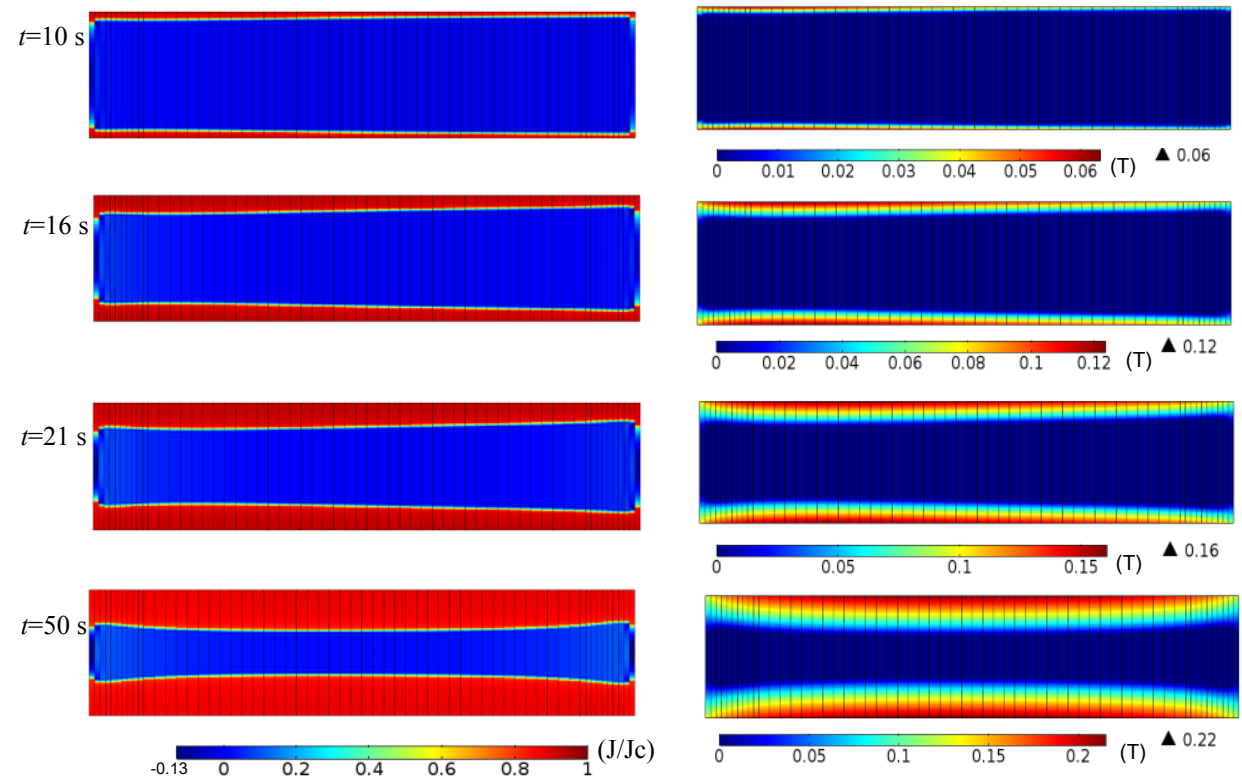

(a) Normalized Azimuthal current density

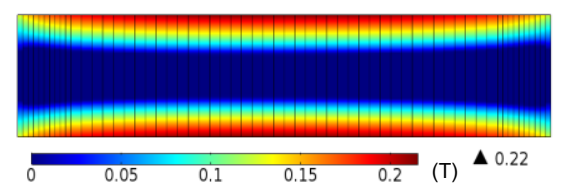

(b) Perpendicular magnetic field

FIG. 9. Left column: distribution of the normalized azimuthal current density; right column: magnetic field perpendicular to the tape surface in the ramping process. 


\section{B. Comparison with conventional INS coil}

In this section, comparison is performed on the NI coil and its insulated counterpart to conduct a further analysis. Identical simulations are performed on the simple NI coil and its insulated counterpart, in which the coil is ramped up to the target current, $20 \mathrm{~A} \sim 100 \mathrm{~A}$, with a ramping rate of $5 \mathrm{~A} / \mathrm{s}$.

Fig. 10 shows the magnetization loss power of the INS coil in the ramping process. The magnetization loss power increases rapidly with the transport current, and reaches to the peak point when the transport current stops increasing, then it drops sharply to zero. The peak value of the INS coil's magnetization loss power shows an exponential increase with the current magnitude, which is induced by the E-J power law of HTS. Fig. 11 shows the turn-to-turn loss power and magnetization loss power of the NI coil. The peak value of the turn-to-turn loss power shows an approximately linear increase with the current magnitude. The NI coil's magnetization loss power is much lower than that of the INS coil because the magnetization loss power of HTS coils depends on the real ramping rate of the azimuthal current in superconductors. With a same ramping rate of power supply, the real ramping rate of the NI coil is much lower than that of its insulated counterpart, due to the ramping delay, as shown in Fig. 5.

Fig. 12 shows the dependence of the loss energy in the whole ramping process on the current magnitude. The NI coil's turn-to-turn loss energy shows an approximately linear increase with the current magnitude. The magnetization losses energy of both the NI and its insulated counterpart increase dramatically with the current magnitude. With a same current magnitude, the magnetization loss energy of the NI coil is almost equal to that of the INS coil, though NI coil's magnetization loss power is much lower than that of the INS coil. The results show that the total magnetization loss energy during a ramping operation only depends on the current magnitude and is independent of the ramping time or raping rate. Since the turn-to-turn loss energy is ten times more than that of the magnetization loss energy, the total ramping loss of the NI coil is also much more than of that of the INS coil.

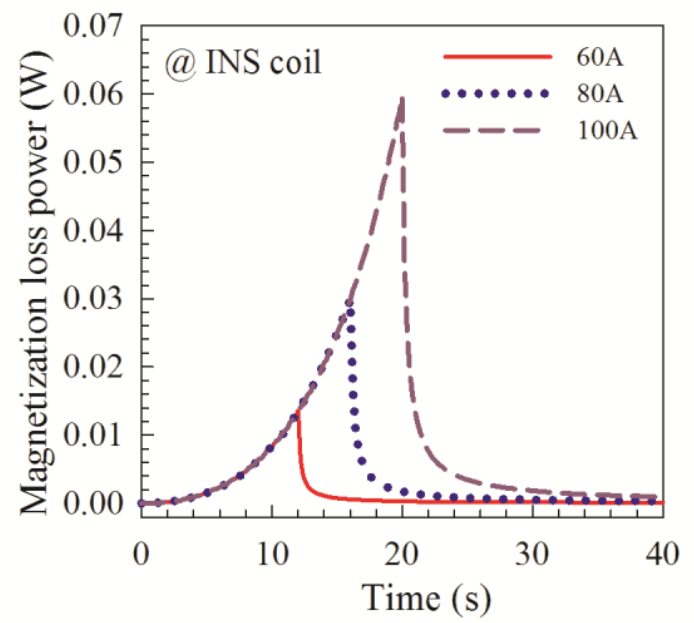

FIG. 10. The magnetization loss power of INS coil in the ramping process, ramping rate $5 \mathrm{~A} / \mathrm{s}$. 

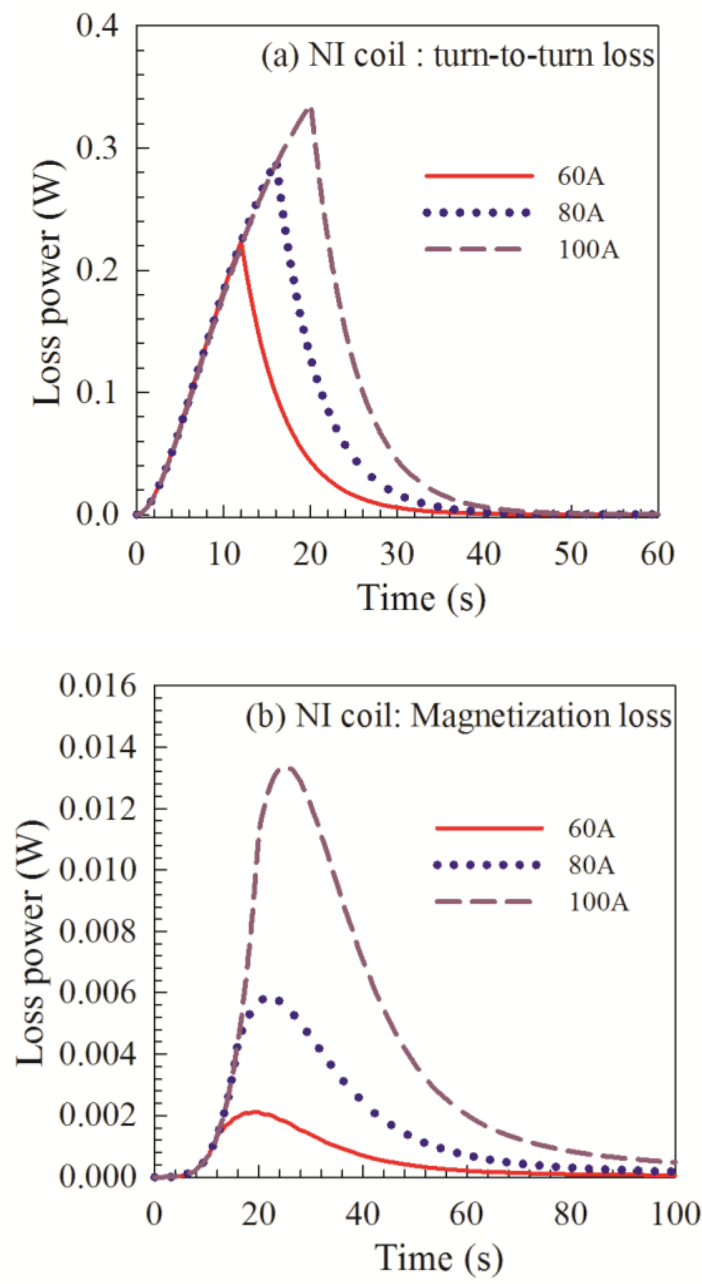

FIG. 11. The magnetization loss power of the NI coil in the ramping process, ramping rate of power supply 5 A/s.

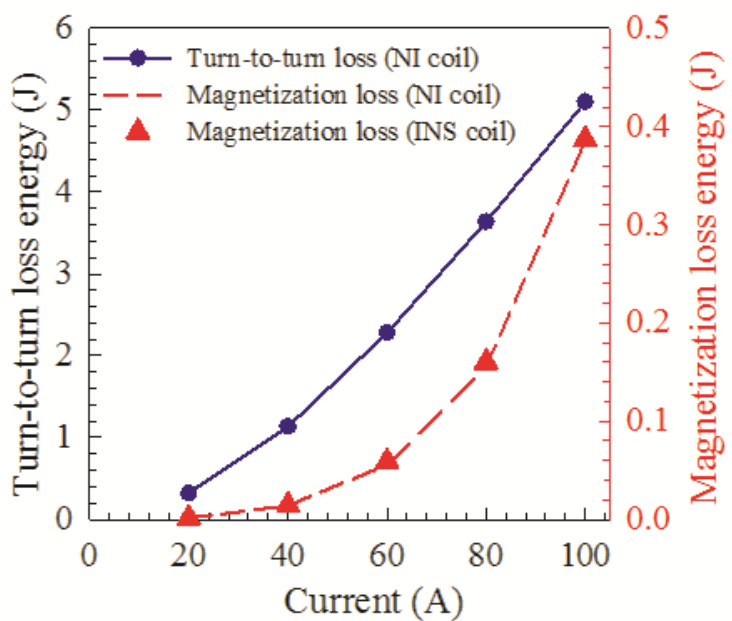

FIG. 12. The dependence of the ramping loss (turn-to-turn loss and magnetization loss) on the current magnitude, ramping rate of power supply $5 \mathrm{~A} / \mathrm{s}$. 


\section{OPTIMIZATION ON NI COIL'S RAMPING LOSS}

Since NI coil's ramping loss is much higher than that of the conventional INS coil, it may be too high to be practical for industrial applications. Therefore, it is necessary to study how to reduce the ramping loss of NI coils, which is conducted in this section by analyzing the factors affecting it: ramping rate and turn-to-turn resistivity.

\section{A. Influence of ramping rate}

The ramping rate is a key factor affecting the ramping losses of conventional INS coils. Here, the "ramping rate" is that of the transport current from the power supply. For INS coils, the transport current always flows along azimuthal current, which is same with that from power supply. For NI coils, the real ramping rate of azimuthal current may be much lower than that of power supply, which depends on the ramping rate of power supply and the coil's time constant.

Simulations are performed on the sample NI coil, in which it is ramped up to $80 \mathrm{~A}$ with different ramping rates. The NI coil's equivalent turn-to-turn resistivity is still set $30 \mu \Omega \cdot \mathrm{cm}^{2}$ in this section. Fig. 13 shows the ramping rate's influence on the turn-to-turn loss power. Higher ramping rate means higher turn-to-turn loss power, because higher ramping rate leads to higher voltage between turns, therefore more radial current flowing through turn-to-turn contacts. Figure 14 shows the dependence of the turn-to-turn loss power peak and total turn-to-turn loss energy in the whole ramping process on the ramping rate of power supply. Both of them increase rapidly with the ramping rate when the ramping is in a low range, but the increasing rate drops gradually. When the ramping rate is high enough, the turn-to-turn loss energy seems to stop increasing and converges to a constant value.

Fig. 15 illustrates the influence of the ramping rate on the magnetization loss power. The higher the ramping rate, the earlier the magnetization loss power increases up to peak. Higher ramping rate means higher magnetization loss power when the ramping rate is in a lower range, then a constant maximum value happens on both the magnetization loss power $W_{s c}$ and energy $Q_{s c}$ when the ramping rate is in a higher rage. As shown in Fig. 16, the magnetization loss power peak increases rapidly with the ramping rate when it is less than $2 \mathrm{~A} / \mathrm{s}$, and then it keeps constant at higher ramping rates. The magnetization loss power of HTS coil depends on the azimuthal-direction current in the superconductor layer and its real ramping rate. When the ramping rate is in a low range, increasing the ramping rate can significantly reduce the time of ramping process, and increase the real ramping rate of the azimuthal current. When the ramping rate is high enough, the total ramping time is determined by the delay process, the total ramping time of NI coil drops to a minimum value ${ }^{19}$. The real ramping rate of azimuthal current converges to a constant value, increasing the ramping rate of power supply cannot accelerate the ramping process. Therefore, the magnetization loss power converges to a constant maximum value. The total magnetization loss energy in the whole ramping process $Q_{s c}$ is independent of the ramping rate. The results show that the turn-to-turn loss may be reduced to the same order of magnitude with the magnetization loss by decreasing the ramping rate, though the turnto-turn loss is 20 times more than the magnetization loss in the above studies. For example, the turn-to-turn loss and magnetization loss are $0.66 \mathrm{~J}$ and $0.16 \mathrm{~J}$ respectively when the ramping rate is $0.5 \mathrm{~A} / \mathrm{s}$; while they are $3.63 \mathrm{~J}$ and 0.17 $\mathrm{J}$ respectively when the ramping rate is $5 \mathrm{~A} / \mathrm{s}$.

As the turn-to-turn loss account for main proportion of the total ramping loss (for low contact resistivity at 30 $\mu \Omega \cdot \mathrm{cm}^{2}$ ), decreasing the ramping rate of power supply can significantly reduce the NI coil's ramping loss, as shown 
in Fig. 17. For the NI coil studied in this section, its total ramping loss energy can be reduced from $3.81 \mathrm{~J}$ to $0.82 \mathrm{~J}$ by decreasing the ramping rate from $5 \mathrm{~A} / \mathrm{s}$ to $0.5 \mathrm{~A} / \mathrm{s}$. However, lower ramping rate always means longer total ramping time required, as shown in Fig. 17. For this sample NI coil, the total ramping time will increase from $54 \mathrm{~s}$ to $185 \mathrm{~s}$. Here the total ramping time here is the time required to charge the coil to generate $99 \%$ of the target magnetic field at coil center. More details about it have been shown in previous publication ${ }^{19}$. The total ramping time of NI coils may be too long to be practical for large-scale applications [15]. An optimization analysis on the ramping rate is required to obtain a balance between ramping time and ramping loss in the design of industrial NI applications. An actively controlled ramping method could also be considered other than the constant ramping rate to minimize the intrinsic ramping delay.

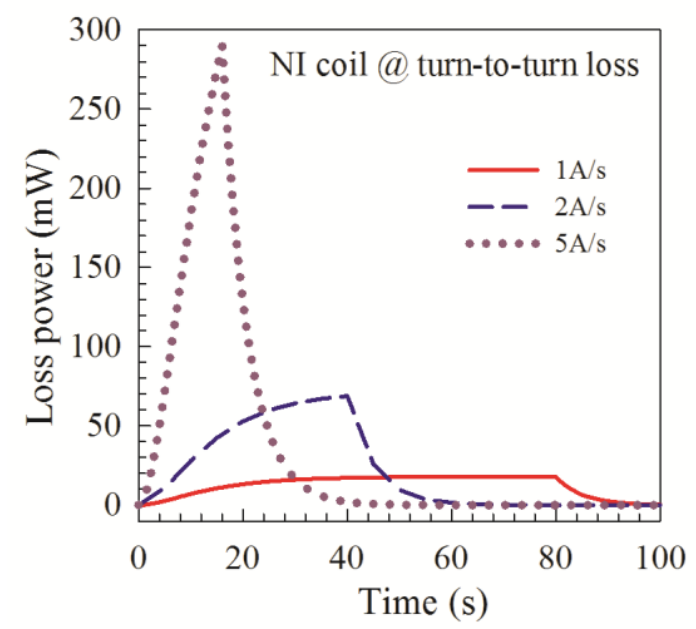

FIG. 13. The turn-to-turn loss power in the ramping process at different ramping rates, $1 \mathrm{~A} / \mathrm{s}, 2 \mathrm{~A} / \mathrm{s}$ and $5 \mathrm{~A} / \mathrm{s}$.

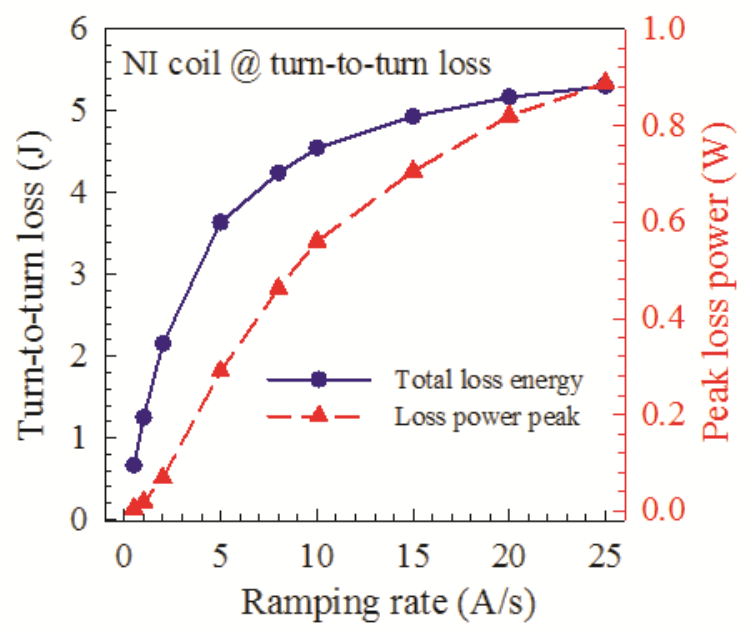

FIG. 14. Dependence of the turn-to-turn loss energy and its power peak on the ramping rate. 


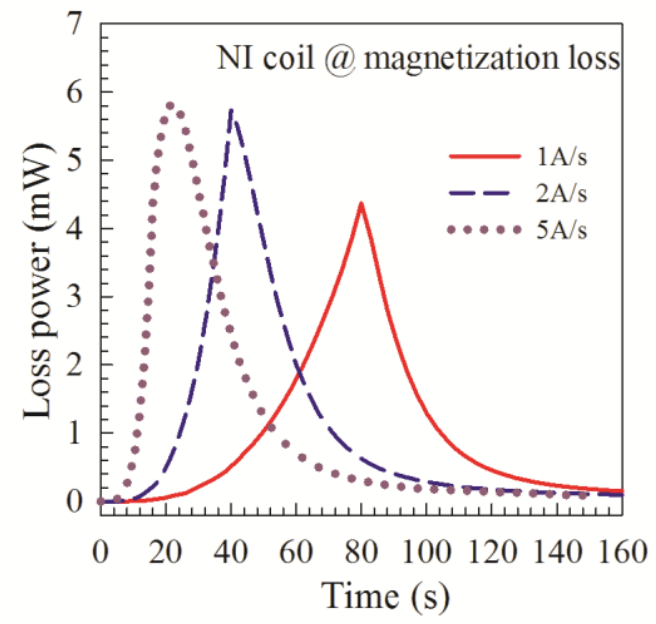

FIG. 15. The magnetization loss power in the ramping process under different ramping rates.

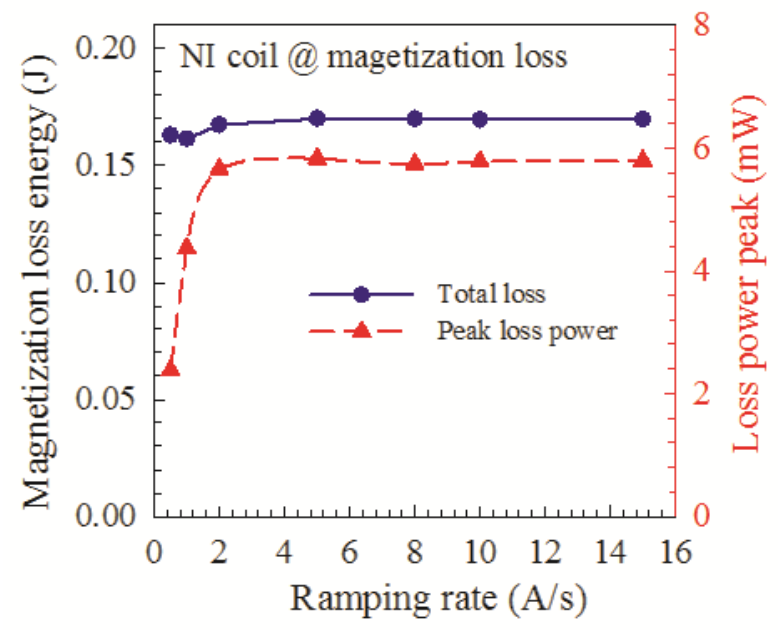

FIG. 16. Dependence of the magnetization loss and its power peak on the ramping rate.

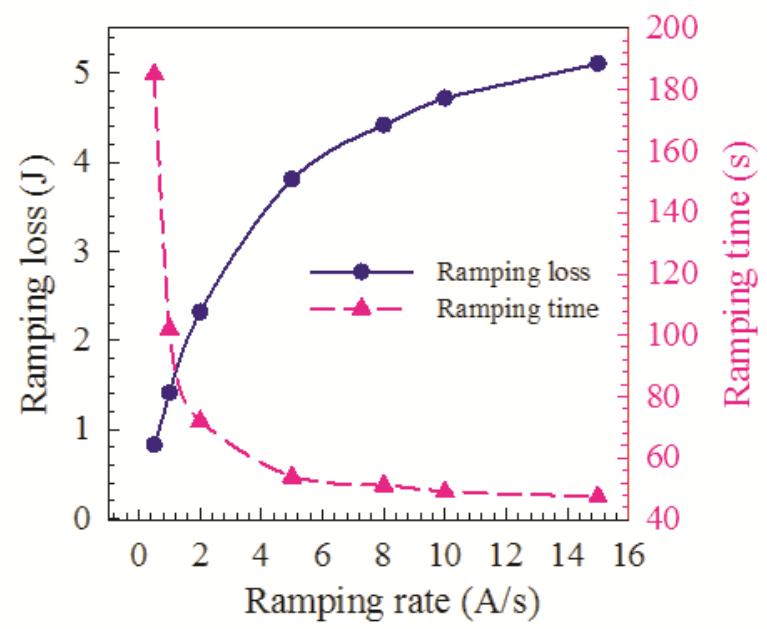

FIG. 17. Dependence of the total ramping loss energy and ramping time on the ramping rate of power supply. 


\section{B. Influence of turn-to-turn resistivity}

The turn-to-turn resistivity is a key parameter of the NI coil, which has a great influence on its ramping behaviors. Its value ranges from $10 \mu \Omega \cdot \mathrm{cm}^{2}$ to $100 \mu \Omega \cdot \mathrm{cm}^{2}$ for NI coils in liquid nitrogen $(77 \mathrm{~K})[5,15$, 40], as well as recently reported by J. Lu et al at the Applied Superconductivity Conference (ASC) $2016^{46}$. More detail about its calculation and measurement has been presented in previous publications ${ }^{4,32,47}$. The turn-to-turn resistivity can be adjusted by changing winding tension, materials and thickness of the tape's metallic layers, contact surface condition, temperature or other factors. Above studies are conducted with a same turn-to-turn resistivity $30 \mu \Omega \cdot \mathrm{cm}^{2}$. This section is to study its influence on the ramping loss of NI coils, which can be an effective method to optimize the ramping loss.

Simulations are performed on the sample NI coils with different turn-to-turn resistivities, during which the coil is ramped up to $80 \mathrm{~A}$ with same ramping rate $(5 \mathrm{~A} / \mathrm{s})$. Fig. 18 shows the influence of turn-to-turn resistivity on the turn-to-turn loss power peak and total loss energy generated during ramping operations. The loss power peak increases rapidly with the increases of turn-to-turn resistivity, and reaches to a peak at some value (about $20 \mu \Omega \cdot \mathrm{cm}^{2}$ in this study), then it drops. The total turn-to-turn loss energy in the whole ramping process drops continually with the increase of the turn-to-turn resistivity. The turn-to-turn loss power is proportional to the turn-to-turn resistance and the square of the radial current, as shown in Eq. (3). Higher turn-to-turn resistivity means less radial current, higher radial resistance and shorter ramping time. These comprehensive influences lead to the trend of the turn-toturn loss in Fig. 18.

Fig. 19 shows the magnetization loss power peak and total loss energy generated in the ramping process with different turn-to-turn resistivity. The loss power peak increases approximately linearly with the turn-to-turn resistivity. Note that the magnetization loss power of HTS coil depends on the actual current insides superconducting layers and its specific ramping rate. Increasing the turn-to-turn resistivity can significantly reduce the ramping delay of NI coils, and thus increase the real ramping rate of the azimuthal transport current in superconductors, which therefore increases the magnetization loss power. However, the total magnetization loss energy in the whole ramping process is independent of the turn-to-turn resistivity, since higher turn-to-turn resistivity also means shorter total ramping time. The results in Fig.12, Fig.16 and Fig. 20 show that the total ramping magnetization loss energy generated in superconductors solely depends on the magnitude of operating current and is independent of the ramping rate and turn-to-turn resistivity.

The above analysis shows that the NI coil's ramping loss can be significantly reduced by increasing the turn-toturn resistivity. However, this may also reduce the thermal stability of the NI coil. During a local quench, higher turn-to-turn resistivity prevents the transport current bypassing to neighboring turns. If turn-to-turn resistivity increases up to infinite, the NI coil will become a conventional INS coil. An optimization on the turn-to-turn resistivity has to be conducted to obtain a balance point between ramping loss and thermal stability in the design of industrial NI HTS applications. 


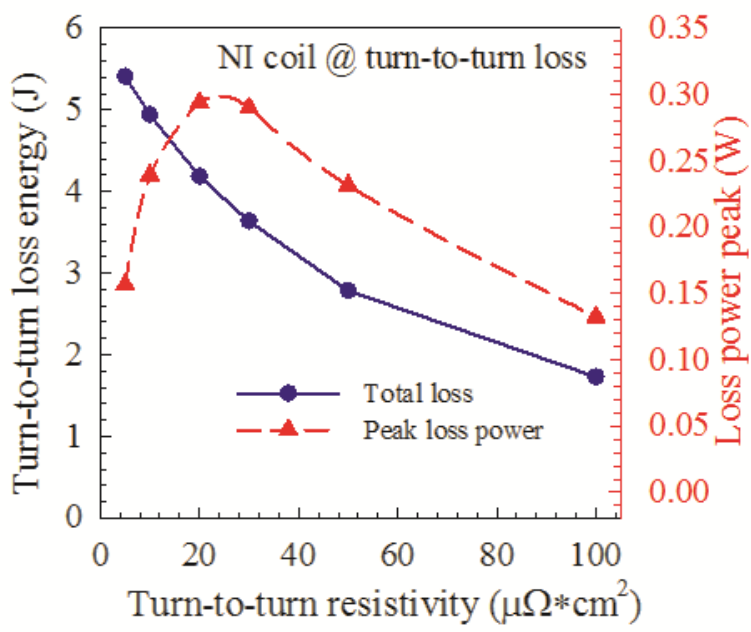

FIG. 18. Dependence of the turn-to-turn loss and its power peak on the turn-to-turn resistivity.

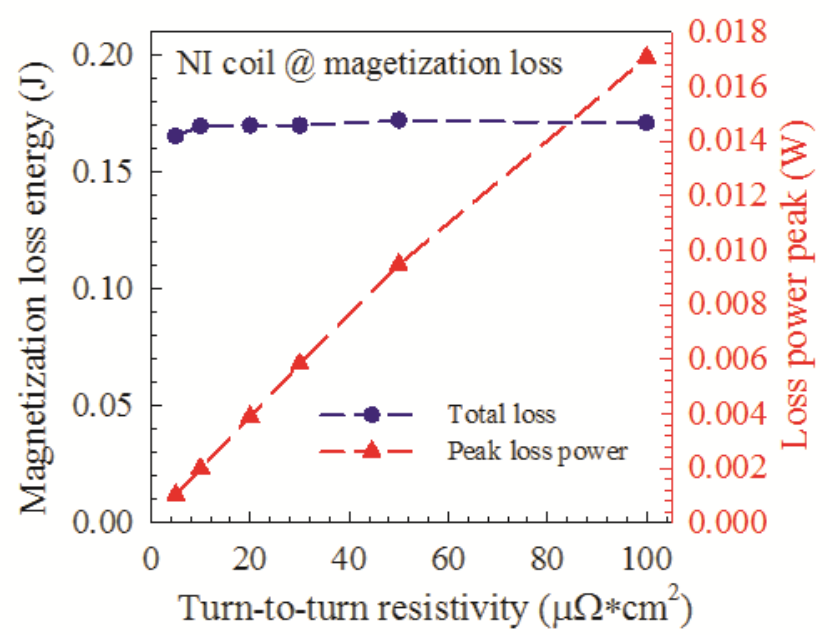

FIG. 19. Dependence of the magnetization loss and its power peak on the turn-to-turn resistivity.

\section{Discussion}

This paper has studied the turn-to-turn loss and magnetization loss of the NI REBCO coil in the ramping process. The magnetization loss is generated in the superconducting layer which is the primary part of the conventional INS coil's AC loss. The turn-to-turn loss is generated by the 'bypassing' radial current on the turn-to-turn contacts of the NI coil, which can be one order of magnitude higher than the according magnetization loss. The magnetization loss energy during the ramping of REBCO coils depends solely on the magnitude of operating current and is independent of the ramping rate and turn-to-turn resistivity. With the same current magnitude, the magnetization loss energy of the NI coil is equal to that of its insulated counterpart. Therefore, the total ramping loss energy of the NI coil is much higher than that of its insulated counterpart, which may be a critical design parameter with regarding to the refrigeration requirements of applications. 


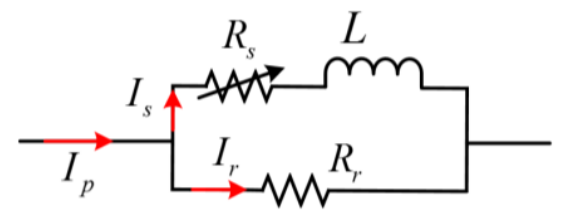

FIG. 20. A lumped equivalent circuit model for NI REBCO coil

Moreover, the magnetization loss is mainly determined by the azimuthal current flowing in superconductors, the turn-to-turn loss is mainly determined by the radial current flowing through turn-to-turn contacts. Both the azimuthal and radial current depend on the ramping rate of power supply and turn-to-turn resistivity. Therefore, the behavior of the NI coil's magnetization loss and turn-to-turn loss is determined by the ramping rate and turn-to-turn resistivity. To understand the behaviors of NI coil's ramping loss with a view of detailed mathematic derivation, a lumped equivalent circuit model for NI REBCO coil shown in Fig. 20 is used, whose governing equation is

$$
\left\{\begin{array}{l}
L \frac{d I_{s}}{d t}+I_{s} R_{s}-I_{r} R_{r}=0 \\
I_{s}+I_{r}=I_{p}(t)
\end{array}\right.
$$

where $L$ represents the coil's self-inductance. $I_{p}, I_{s}$ and $I_{r}$ are the transport current from power supply, azimuthal current in the superconducting layer and radial current on turn-to-turn contacts respectively. $R_{s}$ is the resistance of superconductors, which is nearly zero below the critical current. $R_{r}$ represents the total radial resistance, which is the sum of each turn's radial resistance:

$$
R_{r}=\rho_{t} \sum_{n} \frac{1}{\pi w_{d} D_{k}}
$$

where $\rho_{t}$ is the turn-to-turn resistivity, $w_{d}$ is the tape width, $D_{k}$ is the inner diameter of the $n$-th turn.

When the current of power supply $I_{p}$ is ramped up linearly to the target current $I_{o p}$ with a ramping rate $k$, the solution the solution of this governing equation is:

$$
\begin{aligned}
& I_{s}(t)= \begin{cases}k\left[t-\tau\left(1-e^{-t / \tau}\right)\right] ; & t<t_{r} \\
I_{o p}+k \tau\left(1-e^{I_{o p} / k \tau}\right) e^{-t / \tau} ; & t \geq t_{r}\end{cases} \\
& I_{r}(t)= \begin{cases}k \tau\left(1-e^{-t / \tau}\right) ; & t<t_{r} \\
-k \tau\left(1-e^{t_{r} / \tau}\right) e^{-t / \tau} ; & t \geq t_{r}\end{cases}
\end{aligned}
$$

where:

$$
\tau=\frac{L}{R_{r}}, t_{r}=\frac{I_{o p}}{k}, I_{p}(t)=\left\{\begin{array}{l}
k t ; t<t_{r} \\
I_{o p} ; t \geq t_{r}
\end{array}\right.
$$

More detail about the solution process is included in the Appendix 1.

\section{A. Discussions on turn-to-turn loss}

The turn-to-turn loss power can be derived from formula (16): 


$$
W_{t 2 t}(t)= \begin{cases}k^{2} \tau^{2}\left(1-e^{-t_{r} / \tau}\right)^{2} R_{r} ; & t<t_{r} \\ k^{2} \tau^{2}\left(1-e^{t_{r} / \tau}\right)^{2} e^{-2 t / \tau} R_{r} ; & t \geq t_{r}\end{cases}
$$

The formula (16) and (18) show that the radial current and turn-to-turn loss power always increase up to the peak point at the end of the power supply's ramping process, $t=t_{r}$. The peak value of the radial current and turn-to-turn loss power is:

$$
\left\{\begin{array}{l}
I_{r, \text { peak }}=k \tau\left(1-e^{-t_{r} / \tau}\right) \\
W_{t 2 t, \text { peak }}=k^{2} \tau^{2}\left(1-e^{-t_{r} / \tau}\right)^{2} R_{r}
\end{array}\right.
$$

The Eq. show that higher ramping rate $k$ always means higher radial current, therefore higher turn-to-turn loss power, which has a good agreement with the results in Fig. 14. However, if the ramping rate is high enough, $k \rightarrow \infty$, nearly all the transport current flows along the radial direction at the end of the power supply ramping $t=t_{r}$, as shown in the following Eq. (20). This is the maximum value of the radial current. Therefore, the turn-to-turn loss power peak increases up to a maximum value $I_{o p}{ }^{2} R_{r}$ with the increase of the ramping rate. When the ramping rate $k$ is low enough, $t_{r}$ is much longer than the time constant $\tau$, both the peak radial current and turn-to-turn loss power is approximately zero, which also agree well with the results in Fig. 14.

$$
\lim _{k \rightarrow \infty} I_{r, p e a k}=\lim _{t_{r} \rightarrow 0} \frac{I_{o p}}{t_{r}} \tau\left(1-e^{-t_{r} / \tau}\right)=I_{o p}
$$

When the turn-to-turn resistance $R_{r}$ is high enough, both the radial current and turn-to-turn loss power are nearly zero, as shown in the following Eq. (21). Therefore, the turn-to-turn loss power can be reduced effectively by increasing the turn-to-turn resistivity. However, when the turn-to-turn resistance $R_{r}$ is in a low range, the derivative of $W_{t 2 t}$ peak with respect to $R_{r}$ may be positive, as shown in Eq. (22), which means that the turn-to-turn loss power will increase with the turn-to-turn resistivity in this range. These results agree well with that in Fig. 18. This also explains why the total turn-to-turn loss power has a peak at around $20 \mu \Omega \cdot \mathrm{cm}^{2}$ but the overall turn-to-turn loss energy have a monotonic decrease with increasing resistivity.

$$
\begin{gathered}
\left\{\begin{array}{l}
\lim _{R_{r} \rightarrow \infty} I_{r, \text { peak }}=\lim _{\tau \rightarrow 0} k \tau(1-0)=0 \\
\lim _{R_{r} \rightarrow \infty} W_{t 2 t, \text { peak }}=k^{2} L \lim _{\tau \rightarrow 0} \tau\left(1-e^{-t_{r} / \tau}\right)^{2}=0
\end{array}\right. \\
\frac{\mathrm{d} W_{t 2 t, \text { peak }}}{\mathrm{d} R_{r}}=\frac{k^{2}\left(e^{x}-1\right)\left(2 x-e^{x}+1\right)}{R_{r}^{2} e^{2 x}} ;\left(x=t_{r} R_{r} / L\right)
\end{gathered}
$$




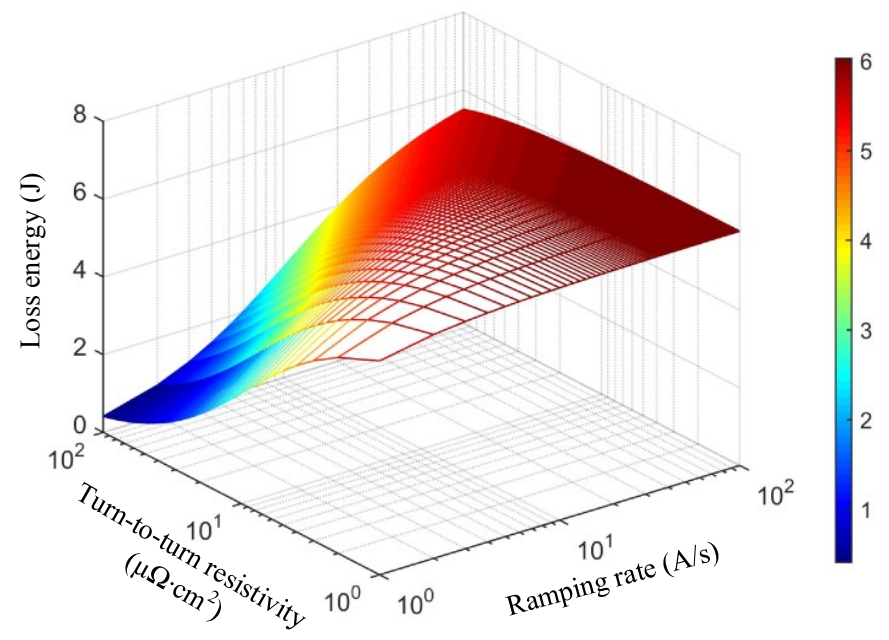

FIG. 21. The two-dimensional dependence of the turn-to-turn loss energy on ramping rate of power supply and turn-to-turn resistivity.

The total turn-to-turn loss energy $Q_{t 2 t}$ generated in the whole ramping process can be derived from Eq. (18):

$$
Q_{t 2 t}=\int W_{r} \mathrm{~d} t=\int_{0}^{t_{r}} W_{r} \mathrm{~d} t+\int_{t_{r}}^{\infty} W_{r} \mathrm{~d} t=k^{2} \tau^{2} R_{r}\left(t_{r}-\tau+\tau e^{-t_{r} / \tau}\right)
$$

For the sample NI coil in Table 1, the dependence of turn-to-turn loss energy on the ramping rate of the power supply and turn-to-turn resistivity can be obtained from this formula, as shown in the Fig. 21. The results show that the turn-to-turn loss energy can always be reduced by decreasing the ramping rate and increasing the turn-to-turn resistivity, which agree well with the results in Fig. 14 and Fig. 18. Furthermore, if the ramping rate is high enough, $k \rightarrow \infty$, or the turn-to-turn resistivity is low enough, $\rho_{t} \rightarrow 0$, the turn-to-turn loss energy seems to converge to a maximum value. The following Eq. (24) also validates this conclusion, its deriving process in shown in Appendix 2. This maximum value is equal to the magnetic energy stored in the coil, which agrees with the results in Fig.14 and Fig. 21. It is an intrinsic characteristics of the NI coil, and has nothing to do with the ramping rate of power supply and turn-to-turn resistivity. This maximum turn-to-turn loss value will be a very useful parameter for the estimation of refrigeration requirements of NI applications.

$$
\left\{\begin{array}{l}
\lim _{k \rightarrow \infty} Q_{t 2 t}=\lim _{t_{r} \rightarrow 0} \tau^{2} R_{r} I_{o p}{ }^{2} \frac{t_{r}-\tau+\tau e^{-t_{r} / \tau}}{t_{r}^{2}}=\frac{1}{2} L I_{o p}^{2} \\
\lim _{\rho_{t} \rightarrow 0} Q_{t 2 t}=\lim _{R_{r} \rightarrow 0} k^{2} L^{2} \frac{t_{r}-\tau+\tau e^{-t_{r} / \tau}}{R_{r}}=\frac{1}{2} L I_{o p}^{2}
\end{array}\right.
$$

\section{B. Discussions on magnetization loss}

The magnetization loss power (time-resolved) of REBCO coils depends on the azimuthal current magnitude and its specific ramping rate. With the same target current, the magnetization loss power increases with the ramping rate of azimuthal current in the superconducting layer. Higher turn-to-turn resistance and higher ramping rate of the 
power supply means high ramping rate of azimuthal current, therefore higher magnetization loss power. However, when the ramping rate of power supply is high enough, $k \rightarrow \infty$; the ramping process of power supply is very short, $t_{r} \rightarrow 0$; almost no transport current flows along the azimuthal direction, $I_{s}\left(t=t_{r}\right) \rightarrow 0$; the increase of the azimuthal current occurs mainly in the following process, $t>t_{r}$. As shown in the Eq. (25), the real ramping rate of azimuthal current in this period is mainly determined by the coil's time constant $\tau$, and has nothing to do with the ramping rate $k$ of power supply. Therefore, the peak magnetization loss power converges to a maximum value with the increase of ramping rate $k$, which agrees well with the results in Fig. 16.

$$
\lim _{k \rightarrow \infty} I_{s}(t)=\lim _{k \rightarrow \infty}\left[I_{o p}+k \tau\left(1-e^{I_{o p} / k \tau}\right) e^{-t / \tau}\right]=I_{o p}\left(1-e^{-t / \tau}\right)
$$

When the turn-to-turn resistivity is high enough, $\rho_{t} \rightarrow \infty$, the coil's time constant is nearly zero, the azimuthal current is approximately equal to the transport current from the power supply, as shown in the Eq. (26). The maximum ramping rate of the azimuthal current in the superconducting layer is the ramping rate $k$ of the power supply, therefore, the magnetization loss power is always less than that of its conventional insulated counterpart.

$$
\lim _{\tau \rightarrow 0} I_{s}(t)= \begin{cases}\lim _{\tau \rightarrow 0} k\left[t-\tau\left(1-e^{-t / \tau}\right)\right]=k t ; & t<t_{r} \\ \lim _{\tau \rightarrow 0}\left[I_{o p}+k \tau\left(1-e^{I_{o p} / k \tau}\right) e^{-t / \tau}\right]=I_{o p} ; & t \geq t_{r}\end{cases}
$$

In addition, as compared in Fig. 10 and 11, their magnetization loss power profiles are much different, and the INS coil's profile is consistent with the power supply current profile and it has a very sharp turning, but the NI coil has some delay and it has a round turning. It could be explained by Fig. 7, 8 and 9, where the power density distributions of the turn-to-turn loss and magnetization loss are much different. When the power supply current reaches its maximum, the radial current starts to transform into the azimuthal current, such redistribution likely occurs to not only two neighboring turns, but rather a macroscopic behavior within all the turns, so it takes some time (though very short) to reach the equilibrium critical current distribution. It likely the reason for the observed $4 \mathrm{~s}$ delay found in Fig. 6 .

\section{CONCULSION}

In this paper, a hybrid numerical method has been developed to calculate the total ramping loss of the NI REBCO pancake coil including both the turn-to-turn loss and magnetization loss and their loss power distributions (time dependent and spatially non-uniform). The model couples the equivalent circuit network model and the $\mathrm{H}$ formulation based FEM model. The ramping loss of the NI coil consists of the turn-to-turn loss because of the existing radial current and the magnetization loss related to the flux creep and flux jump in the REBCO superconductors. The equivalent circuit network model has calculated the turn-to-turn loss and the FEM model has calculated the magnetization loss. A DP NI coil is wound by REBCO tapes and this numerical method is validated by the experiments. Then the characteristics of the NI coil's ramping loss are studied using the models. It has achieved quite a few conclusions as follows.

Regarding the NI HTS coils, the turn-to-turn loss can be one order of magnitude higher than the magnetization loss. The total ramping loss of the NI coil during a ramping operation significantly depends on the turn-to-turn loss. 
With the assumption of uniform turn-to-turn resistivity among turns, both the turn-to-turn loss and magnetization loss shows a non-uniform distribution among turns, the maximum value may be two times larger than the minimum value. In fact, the actual resistivity between turns may be not constant which actually depends on the accumulated pressures in the HTS coils, and further study is needed.

Secondly, during the same ramping operation (the same current magnitude and ramping rate of the power supply), the NI coil's magnetization loss power is much lower than that of its insulated counterpart, due to significant ramping delay. The magnetization loss power can be reduced by decreasing the ramping rate of power supply and increasing the turn-to-turn resistivity, due to less ramping delay. However, the magnetization loss energy generated in the whole ramping process depends solely on the magnitude of operating current and is independent of the ramping rate and turn-to-turn resistivity. Overall, the total ramping loss energy of the NI coil is much higher than that of its insulated counterpart, due to turn-to-turn loss.

Thirdly, higher ramping rate of power supply always means higher turn-to-turn loss power and more turn-to-turn loss energy generated in the whole ramping process. The maximum turn-to-turn loss power happens when almost all the transport current flows along radial direction. The higher turn-to-turn resistivity means higher turn-to-turn loss power when it is in a low range, then the turn-to-turn loss power drops with the increase of turn-to-turn resistivity. Higher ramping rate of power supply and lower turn-to-turn resistivity always mean more turn-to-turn loss energy generated in the whole ramping process. The maximum turn-to-turn loss energy is equal to the magnetic energy stored in the coil, which happens when the ramping rate is high enough or the turn-to-turn resistivity is low enough.

Furthermore, the total ramping loss of NI coils can be reduced significantly reduced by decreasing the ramping rate and increasing the turn-to-turn resistivity. However, lower the ramping rate means longer ramping time, and higher turn-to-turn resistivity may reduce the thermal stability though a self-protection feature of the NI coil does exist and benefit. As such, multiple parameters optimization is required on the total ramping loss, total ramping time and thermal stability to obtain an optimal point in the design of NI HTS applications.

\section{ACKNOWLEDGMENTS}

This work was sponsored by National Natural Science Foundation of China, "Modeling and experimental study on the electromagnetic and thermal characteristics of no-insulation high temperature superconductor coils", Project No. 51577119.

The authors would like to thank the Key Laboratory of Control of Power Transmission and Conversion (Ministry of Education), The Shanghai Engineering Center for Superconducting Materials and System, and the State Energy Smart Grid R\&D Center (Shanghai) for the support on assistance in experiments. 


\section{REFERENCES}

1. Y. Iwasa, J. Bascuan, S. Hahn, M. Tomita and Y. Weijun, IEEE Appl. Supercond. 20 (3), 718-721 (2010).

2. J. Schwartz, T. Effio, L. Xiaotao, Q. V. Le, A. L. Mbaruku, H. J. Schneider-Muntau, S. Tengming, S. Honghai, U. P. Trociewitz, W. Xiaorong and H. W. Weijers, IEEE Appl. Supercond. 18 (2), 70-81 (2008).

3. H. Song, P. Brownsey, Z. Yifei, J. Waterman, T. Fukushima and D. Hazelton, IEEE Appl. Supercond. 23 (3), 4600806 (2013).

4. S. Hahn, D. K. Park, J. Bascunan and Y. Iwasa, IEEE Appl. Supercond. 21 (3), 1592-1595 (2011).

5. S. Hahn, D. K. Park, K. Kim, J. Bascunan and Y. Iwasa, IEEE Appl. Supercond. 22 (3) (2012).

6. A. V. G. Dudarev, A. V.; Ilyin, Yu. A. ; Keilin, V.E.; Kopeikin, N. Ph.; Shchrbakov, V.I.; Shugaev, I.O. and Stepanov, V.V. , in Inst. Phys. Conference Ser. No 158 (Pro. EUCAS-97) (1997), pp. 1615-1619.

7. Y. H. Choi, O. J. Kwon, Y. G. Kim, J. B. Song, J. H. Kim, H. M. Kim and H. Lee, IEEE Appl. Supercond. 24 (3), 5 (2014).

8. W. D. Markiewicz, J. J. Jaroszynski, D. V. Abraimov, R. E. Joyner and A. Khan, Supercond. Sci. Technol. 29 (2), 25001-25001 (2016).

9. Y. Sangwon, K. Jaemin, L. Hunju, H. Seungyong and M. Seung-Hyun, Supercond. Sci. Technol. 29 (4), 04LT04 (2016).

10. J.-B. Song, S. Hahn, T. Lecrevisse, J. Voccio, J. Bascunan and Y. Iwasa, Supercond. Sci. Technol. 28 (11) (2015).

11. Y. Wang, C. Wan Kan and J. Schwartz, Supercond. Sci. Technol. 29 (4), 045007 (2016).

12. S. Choi, H. C. Jo, Y. J. Hwang, S. Hahn and T. K. Ko, IEEE Appl. Supercond. 22 (3) (2012).

13. S. Hahn, Y. Kim, J. Song, J. Voccio, J. Ling, J. Bascunan and Y. Iwasa, IEEE Appl. Supercond. 24 (3) (2014).

14. Y. Iwasa and S. Hahn, Appl. Phys. Lett. 103 (25) (2013).

15. J. Schwartz, Supercond. Sci. Technol. 29 (5) (2016).

16. S. Hahn, Y. Kim, J. Ling, J. Voccio, D. K. Park, J. Bascunan, H.-J. Shin, H. Lee and Y. Iwasa, IEEE Appl. Supercond. 23 (3) (2013).

17. Y. J. Hwang, S. Choi, H. C. Jo, Y. H. Choi, T. J. Kim, S. Hahn and T. K. Ko, IEEE Appl. Supercond. 23 (3) (2013).

18. K. L. Kim, Y. H. Choi, D. G. Yang, J. B. Song and H. G. Lee, Supercond. Sci. Technol. 27 (1) (2014).

19. Y. Wang and H. Song, Supercond. Sci. Technol. 29 (7), 075006 (2016).

20. W. Yawei, X. Deqiang, S. Hao, L. Xu, S. Jie, L. Ke, H. Zhiyong, J. Zhijian and L. Zhuyong, IEEE Appl. Supercond. 25 (3), 4600305 (2015).

21. S. Jung-Bin, H. Seungyong, K. Youngjae, D. Miyagi, J. Voccio, J. Bascunan, L. Haigun and Y. Iwasa, IEEE Appl. Supercond. 25 (3), 5202905 (2015).

22. Z. Ang, I. Bejar, L. Bottura, D. Richter, M. Sheehan, L. Walckiers and R. Wolf, IEEE Appl. Supercond. 9 (2), $742-745$ (1999).

23. A. R. Burgers and J. A. Eikelboom, IEEE Transactions on Magnetics 28 (1), 850-853 (1992).

24. F. Grilli and S. P. Ashworth, Supercond. Sci. Technol. 20 (8), 794-799 (2007).

25. J. Lu, E. S. Choi, H. Kandel, D. V. Abraimov and W. D. Markiewicz, IEEE Appl. Supercond. 24 (3) (2014).

26. J. J. Rabbers, A. Dudarev, R. Pengo, C. Berriaud and H. H. J. ten Kate, IEEE Appl. Supercond. 16 (2), $549-552$ (2006).

27. M. D. Sumption, E. W. Collings, R. M. Scanlan and A. Nijhuis, IEEE Appl. Supercond. 13 (2), 2376-2379 (2003).

28. M. Zhang, W. Yuan, J. Kvitkovic and S. Pamidi, Supercond. Sci. Technol. 28 (11) (2015).

29. K. Ryu, B. J. Choi and Y. H. Chun, IEEE Appl. Supercond. 13 (2), 2360-2363 (2003).

30. G. Escamez, A. Badel, P. Tixador, B. Ramdane, G. Meunier, A. Allais and C. E. Bruzek, IEEE Appl. Supercond. 25 (3) (2015).

31. W. Yuan, A. M. Campbell and T. A. Coombs, Supercond. Sci. Technol. 22 (7) (2009).

32. Y. Wang, H. Song, D. Xu, Z. Y. Li, Z. Jin and Z. Hong, Supercond. Sci. Technol. 28 (4) (2015).

33. S. Noguchi, R. Itoh, S. Hahn and Y. Iwasa, IEEE Appl. Supercond. 24 (3) (2014).

34. W. Tao, S. Noguchi, W. Xudong, I. Arakawa, K. Minami, K. Monma, A. Ishiyama, H. Seungyong and Y. Iwasa, IEEE Appl. Supercond. 25 (3), 4603409 (2015)

35. Z. Hong, A. M. Campbell and T. A. Coombs, Supercond. Sci. Technol. 19 (12), 1246-1252 (2006).

36. Z. Hong, Q. Jiang, R. Pei, A. M. Campbell and T. A. Coombs, Supercond. Sci. Technol. 20 (4), 331-337 (2007).

37. K. Kajikawa, T. Hayashi, R. Yoshida, M. Iwakuma and K. Funaki, IEEE Appl. Supercond. 13 (2), 3630-3633 (2003).

38. J. Rhyner, Physica C 212 (3-4), 292-300 (1993).

39. C. M. Barth, G.; and Senatore, C.; Supercond. Sci. Technol. 28, 045011 (2015).

40. F. Grilli, F. Sirois, V. M. R. Zermeno and M. Vojenciak, IEEE Appl. Supercond. 24 (6) (2014)

41. V. M. R. Zermeno, A. B. Abrahamsen, N. Mijatovic, B. B. Jensen and M. P. Sorensen, J Appl Phys 114 (17) (2013).

42. V. M. R. Zermeno and F. Grilli, Supercond. Sci. Technol. 27 (4) (2014).

43. L. Queval, V. M. R. Zermeno and F. Grilli, Supercond. Sci. Technol. 29 (2) (2016)

44. W. Yuan, M. D. Ainslie, W. Xian, Z. Hong, Y. Chen, Y. Y., R. Pei and T. A. Coombs, IEEE Appl. Supercond. 21 (3), 2441 (2011).

45. J. Šouc, Pardo, M. Vojenciak and F. Gömöry, Supercond. Sci. Technol. 22 (1), 015006 (2008).

46. J. Lu, R. Goddard, K. Han, and S. Hahn, Supercond. Sci. Technol. 30 (4), 045005 (2017)

47. X. Wang, S. Hahn, Y. Kim, J. Bascunan, J. Voccio, H. Lee and Y. Iwasa, Supercond. Sci. Technol. 26 (3) (2013). 


\section{Appendix 1}

The governing equations of the lumped circuit model:

$$
\left\{\begin{array}{l}
L \frac{d I_{s}}{d t}-I_{r} R_{r}=0 \\
I_{s}+I_{r}=I(t)
\end{array}\right.
$$

Set: $\frac{d I(t)}{d t}=k ; \tau=\frac{R_{r}}{L}$, the above equation can be rewrote as:

$$
\frac{d I_{s}}{d t}+\frac{I_{s}}{\tau}=\frac{I(t)}{\tau}
$$

This is a typical one order linear differential equation, as followings:

$$
\frac{d y}{d x}+p(x) y=Q(x)
$$

This equation's analytical solution is:

$$
y=e^{-\int p(x) d x}\left(\int Q(x) e^{\int p(x) d x} d x+C\right)
$$

Therefore, the analytical solution of the equation (3) is:

$$
I_{s}(t)=e^{-\int \frac{d t}{\tau}}\left(\int \frac{I(t)}{\tau} e^{\int \frac{d t}{\tau}} d t+C\right)=e^{-t / \tau}\left(\int \frac{I(t)}{\tau} e^{t / \tau} d t+C\right)
$$

where $\mathrm{C}$ is unknown constant.

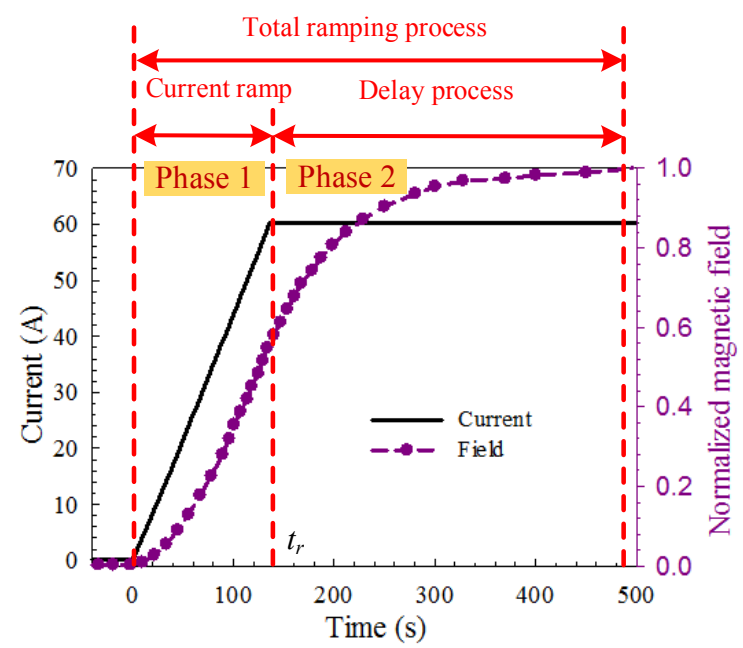

FIG. 1. The transport current from power supply and magnetic field induced at coil center in the ramping process of NI HTS coil.

The whole charging process of NI HTS coils can be subdivided into two phases, as shown in following figure 1.

(1) Phase 1: current ramp process: the transport current from power supply is ramped up to the target current $I_{o p}$ linearly:

$$
I=0 \rightarrow I_{o p} ; I(t)=k t ; d I / d t=k
$$

(2) Phase 2: delay process: the transport current is kept at the target current:

$$
I=I_{o p} ; d I / d t=0
$$

Therefore, the analytical solution Eq. 4 has also to be subdivided into two phases: 
Phase 1: $I=0 \sim I_{o p} ; I(t)=k t ; d I / d t=k$

$$
\begin{aligned}
& I_{s}(t)=e^{-t / \tau}\left(\int \frac{I(t)}{\tau} e^{t / \tau} d t+C\right)=e^{-t / \tau}\left(\frac{k}{\tau} \int t e^{t / \tau} d t+C\right) \\
& =e^{-t / \tau}\left(k\left(t e^{t / \tau}-\int e^{t / \tau} d t\right)+C\right)=e^{-t / \tau}\left(k t e^{t / \tau}-k \tau e^{t / \tau}+C\right) \\
& =k(t-\tau)+C e^{-t / \tau}
\end{aligned}
$$

$t=0, \quad I_{s}(t=0)=0, I(t=0)=0 ; \quad I_{s}(t)=-k / \tau+C=0 \Rightarrow C=k \tau ;$

so:,

$$
\left\{\begin{aligned}
I_{s}(t) & =k(t-\tau)+k \tau e^{-t / \tau}=k\left[t-\tau\left(1-e^{-t / \tau}\right)\right]=\frac{d I}{d t}\left[t-\tau\left(1-e^{-t / \tau}\right)\right] \\
I_{r}(t) & =I(t)-\frac{d I}{d t}\left[t-\tau\left(1-e^{-t / \tau}\right)\right]=k t-k\left[t-\tau\left(1-e^{-t / \tau}\right)\right] \\
& =k \tau\left(1-e^{-t / \tau}\right)=\frac{d I}{d t} \tau\left(1-e^{-t / \tau}\right)
\end{aligned}\right.
$$

Therefore, the solution of governing equation (1) in Phase 1 is:

$$
\left\{\begin{array}{l}
I_{s}(t)=k\left[t-\tau\left(1-e^{-t / \tau}\right)\right] \\
I_{r}(t)=k \tau\left(1-e^{-t / \tau}\right)
\end{array}\right.
$$

Phase 2: $I=I_{o p} ; d I / d t=0$;

In this process, the analytical solution of equation 4 is:

$$
\begin{aligned}
& I_{s}(t)=e^{-t / \tau}\left(\int \frac{I(t)}{\tau} e^{t / \tau} d t+C\right)=e^{-t / \tau}\left(\int \frac{I_{o p}}{\tau} e^{t / \tau} d t+C\right) \\
& =e^{-t / \tau}\left(I_{o p} e^{t / \tau}+C\right)=I_{o p}+C e^{-t / \tau}
\end{aligned}
$$

The end of Phase 1 is the just the initial of the Phase 2, so.

$$
\begin{aligned}
& I_{s}\left(t=\frac{I_{o p}}{k}\right)=k\left[\frac{I_{o p}}{k}-\tau\left(1-e^{-I_{o p} / k \tau}\right)\right]=I_{o p}-k \tau\left(1-e^{-I_{o p} / k \tau}\right) \\
& I_{s}\left(t=\frac{I_{o p}}{k}\right)=I_{o p}+C e^{-I_{o p} / k \tau}=I_{o p}-k \tau\left(1-e^{-I_{o p} / k \tau}\right) \\
& \Rightarrow C=\frac{-k \tau\left(1-e^{-I_{o p} / k \tau}\right)}{e^{-I_{o p} / k \tau}}=k \tau\left(1-e^{I_{o p} / k \tau}\right)
\end{aligned}
$$

So the solution of governing equation (1) in Phase 2 is

$$
I_{s}(t)=I_{o p}+C e^{-t / \tau}=I_{o p}+k \tau\left(1-e^{I_{o p} / k \tau}\right) e^{-t / \tau}
$$

Therefore, the analytical solution of the governing equation (1) is: 


$$
\begin{gathered}
I_{s}(t)= \begin{cases}k\left[t-\tau\left(1-e^{-t / \tau}\right)\right] ; & t<t_{r} \\
I_{o p}+k \tau\left(1-e^{I_{o p} / k \tau}\right) e^{-t / \tau} ; & t \geq t_{r}\end{cases} \\
I_{r}(t)= \begin{cases}k \tau\left(1-e^{-t / \tau}\right) ; & t<t_{r} \\
-k \tau\left(1-e^{I_{o p} / k \tau}\right) e^{-t / \tau} ; & t \geq t_{r}\end{cases}
\end{gathered}
$$

\section{Appendix 2}

$$
\begin{aligned}
W_{r} & =I_{r}^{2} R_{r} \\
W_{r} & =\left\{\begin{array}{l}
k^{2} \tau^{2}\left(1-e^{-t / \tau}\right)^{2} R_{r}, \quad t<t_{r}=I_{o p} / k \\
k^{2} \tau^{2}\left(1-e^{t_{r} / \tau}\right)^{2} e^{-2 t / \tau} R_{r}, \quad t \geq t_{r}=I_{o p} / k
\end{array}\right. \\
Q_{t 2 t} & =\int W_{r} \mathrm{~d} t=\int_{0}^{t_{r}} W_{r} \mathrm{~d} t+\int_{t_{r}}^{\infty} W_{r} \mathrm{~d} t \\
& =k^{2} \tau^{2} R_{r} \int_{0}^{t_{r}}\left(1+e^{-2 t / \tau}-2 e^{-t / \tau}\right) \mathrm{d} t+k^{2} \tau^{2} R_{r}\left(1-e^{-t_{r} / \tau}\right)^{2} \int_{t_{r}}^{\infty} e^{-2 t / \tau} \mathrm{d} t \\
& =k^{2} \tau^{2} R_{r}\left[t_{r}+\frac{\tau}{2}\left(1-e^{-2 t_{r} / \tau}\right)-2 \tau\left(1-e^{-t_{r} / \tau}\right)+\left(1+e^{2 t_{r} / \tau}-2 e^{t_{r} / \tau}\right) \frac{\tau}{2} \lim \left(e^{-2 t_{r} / \tau}-e^{-2 x / \tau}\right)\right] \\
& =k^{2} \tau^{2} R_{r}\left[t_{r}+\frac{\tau}{2}\left(1-e^{-2 t_{r} / \tau}\right)-2 \tau\left(1-e^{-t_{r} / \tau}\right)+\frac{\tau}{2}\left(1+e^{2 t_{r} / \tau}-2 e^{t_{r} / \tau}\right) e^{-2 t_{r} / \tau}\right] \\
& =k^{2} \tau^{2} R_{r}\left[t_{r}+\frac{\tau}{2}-\frac{\tau}{2} e^{-2 t_{r} / \tau}-2 \tau+2 \tau e^{-t_{r} / \tau}+\frac{\tau}{2} e^{-2 t_{r} / \tau}+\frac{\tau}{2}-\tau e^{-t_{r} / \tau}\right] \\
& =k^{2} \tau^{2} R_{r}\left(t_{r}-\tau+\tau e^{-t_{r} / \tau}\right)
\end{aligned}
$$

So:

$$
Q_{t 2 t}=k^{2} \tau^{2} R_{r}\left(t_{r}-\tau+\tau e^{-t_{r} / \tau}\right)
$$

$$
\begin{gathered}
\lim _{k \rightarrow \infty} Q_{t 2 t}=\lim _{t_{r} \rightarrow 0} Q_{t 2 t}=\lim _{t_{r} \rightarrow 0} \tau^{2} R_{r} I_{o p}{ }^{2} \frac{t_{r}-\tau+\tau e^{-t_{r} / \tau}}{t_{r}^{2}} \\
\begin{array}{l}
\text { l'Hopital's rule } \\
=
\end{array} \tau^{2} I_{o p}^{2} R_{r} \lim _{t_{r} \rightarrow 0} \frac{1-e^{-t_{r} / \tau}}{2 t_{r}} \stackrel{\text { l'Hôpital's rule }}{=} \tau^{2} I_{o p}^{2} R_{r} \lim _{t_{r} \rightarrow 0} \frac{t_{r} / \tau}{2 t_{r}} \\
=\frac{\tau}{2} I_{o p}{ }^{2} R_{r}=\frac{L}{2 R_{r}} I_{o p}{ }^{2} R_{r}=\frac{1}{2} L I_{o p}^{2}
\end{gathered}
$$

$$
\begin{gathered}
\lim _{\rho_{t} \rightarrow 0} Q_{t 2 t}=\lim _{R_{r} \rightarrow 0} Q_{t 2 t}=\lim _{R_{r} \rightarrow 0} k^{2} L^{2} \frac{t_{r}-\tau+\tau e^{-t_{r} / \tau}}{R_{r}}=\lim _{R_{r} \rightarrow 0} k^{2} L^{2} \frac{t_{r} R_{r}-L+L e^{-t_{r} R_{r} / L}}{R_{r}^{2}} \\
\begin{array}{c}
l^{\prime} \text { Hôpital's rule } \\
=
\end{array} \lim _{R_{r} \rightarrow 0} k^{2} L^{2} \frac{t_{r}\left(1-e^{-t_{r} R_{r} / L}\right)}{2 R_{r}} \stackrel{\text { l'Hôpital's rule }}{=} \lim _{R_{r} \rightarrow 0} k^{2} L^{2} \frac{t_{r}^{2} R_{r} / L}{2 R_{r}} \\
=k^{2} L^{2} \frac{t_{r}^{2}}{2 L}=\frac{I_{o p}^{2}}{t_{r}^{2}} L^{2} \frac{t_{r}^{2}}{2 L}=\frac{1}{2} I_{o p}^{2} L
\end{gathered}
$$


